\title{
On surfactant-enhanced spreading and superspreading of liquid drops on solid surfaces
}

\author{
GEORGE KARAPETSAS ${ }^{1}$, RICHARD V. CRASTER \\ AND OMAR K. MATAR ${ }^{1} \dagger$ \\ ${ }^{1}$ Department of Chemical Engineering, Imperial College London, South Kensington Campus, \\ London SW7 2AZ, UK \\ ${ }^{2}$ Department of Mathematics, Imperial College London, South Kensington Campus, \\ London SW7 2AZ, UK
}

(Received 14 April 2010; revised 5 August 2010; accepted 14 October 2010; first published online 25 January 2011)

The mechanisms driving the surfactant-enhanced spreading of droplets on the surface of solid substrates, and particularly those underlying the superspreading behaviour sometimes observed, are investigated theoretically. Lubrication theory for the droplet motion, together with advection-diffusion equations and chemical kinetic fluxes for the surfactant transport, leads to coupled evolution equations for the drop thickness, interfacial concentrations of surfactant monomers and bulk concentrations of monomers and micellar, or other, aggregates. The surfactant can be adsorbed on the substrate either directly from the bulk monomer concentrations or from the liquid-air interface through the contact line. An important feature of the spreading model developed here is the surfactant behaviour at the contact line; this is modelled using a constitutive relation, which is dependent on the local surfactant concentration. The evolution equations are solved numerically, using the finite-element method, and we present a thorough parametric analysis for cases of both insoluble and soluble surfactants with concentrations that can, in the latter case, exceed the critical micelle, or aggregate, concentration. The results show that basal adsorption of the surfactant plays a crucial role in the spreading process; the continuous removal of the surfactant that lies upon the liquid-air interface, due to the adsorption at the solid surface, is capable of inducing high Marangoni stresses, close to the droplet edge, driving very fast spreading. The droplet radius grows at a rate proportional to $t^{a}$ with $a=1$ or even higher, which is close to the reported experimental values for superspreading. The spreading rates follow a non-monotonic variation with the initial surfactant concentration also in accordance with experimental observations. An accompanying feature is the formation of a rim at the leading edge of the droplet. In some cases, the drop spreads to form a 'pancake' or creates a 'secondary' front separated from the main droplet.

Key words: contact lines, drops, interfacial flows (free surface)

\section{Introduction}

The spreading of fluids over liquid and solid substrates has attracted the interest of many researchers in the past because of its numerous practical applications and

$\dagger$ Email address for correspondence: o.matar@imperial.ac.uk 
scientific challenges (see, for example, the reviews by de Gennes 1985 and Bonn et al. 2009 and the references therein). It is well known that the addition of a surface-active material (a surfactant) can have a dramatic effect on the rate, extent and uniformity of the spreading process. The ability to control these properties can be very beneficial in many industrial as well as biomedical applications such as coating processes, spraying herbicides (Knoche, Tamura \& Bukovac 1991) and surfactant replacement therapy (Grotberg 1994). In order to be able to optimize the use of surfactants in such applications, however, we have to fully understand their role in the spreading process.

Over many years, researchers have examined the spreading of pure liquid drops on solid surfaces experimentally as well as theoretically (see, for instance, Dussan \& Davis 1974; Greenspan 1978; Tanner 1979; Cox 1986a; Dussan, Rame \& Garoff 1991; Haley \& Miksis 1991; Ehrhard 1993; Carlson, Do-Quang \& Amberg 2009). Recently, renewed interest in the properties of surfactants, and their applications, has led many researchers to investigate surfactant-enhanced spreading, performing experiments for a wide variety of surfactants and substrates (Zhu et al. 1994; Stoebe et al. 1996, 1997a,b,c; Nikolov et al. 2002; Rafai et al. 2002; Radulovic, Sefiane \& Shanahan 2009). The spreading characteristics of surfactants are broadly categorized as follows: (a) surfactants are able to dramatically lower the surface tension of the interfaces upon which they lie, and their mixtures can effectively wet hydrophobic surfaces; $(b)$ the spreading rate becomes maximum on intermediate energy substrates; $(c)$ a maximum in the spreading rate is observed as a function of the initial surfactant concentration; (d) the final wetted area is proportional to the initial concentration of the surfactant. A striking and intriguing experimental observation is that a certain class of surfactants (siloxane-based surfactants) have a unique ability to promote very rapid spreading on highly hydrophobic surfaces. This surfactant-enhanced spreading phenomenon is called 'superspreading', and the relevant surfactants are called superspreaders (Hill 1998, 2002). Despite the widespread attention received by this phenomenon, the mechanism underlying superspreading is not yet fully understood.

Surfactant-enhanced spreading has been attributed by several researchers to the Marangoni effect, which is driven by surface tension gradients, because the advancement of the flow front in many cases follows a $t^{a}$ power law with $a=1 / 2$. However, it has been suggested that Marangoni flow cannot provide a sufficient explanation for the superspreading phenomenon for which spreading rates are often much higher and a power law exponent of $a=1$ may be encountered. Some attribute this increase in the spreading rates simply to a large affinity of the surfactant molecules for the solid substrate or a higher transport efficiency of the superspreaders to the same substrate (Rafai et al. 2002). Others claim that there may be direct adsorption of micelles at the air-liquid and solid-liquid interfaces which provides a much faster interfacial adsorption of the surfactant in order to counterbalance the dilation of the interface (Kumar, Couzis \& Maldarelli 2003).

To clearly elucidate the spreading process and provide possible explanations for experimental observations, theoretical studies have been pursued. Modelling the spreading of pure liquid drops on solid surfaces is challenging, and the physics governing the motion of the contact line still remains unclear. It is well known that when the usual no-slip condition is applied at the liquid-solid interface, a nonintegrable stress singularity appears at the moving contact line (Huh \& Scriven 1971), which has a number of 'remedies' in the literature (Bonn et al. 2009). One involves relaxing the usual no-slip condition at the solid surface; another approach postulates the presence of an 'inner' region close to the contact line, where other mechanisms replace the usual no-slip condition, and an 'outer', or macroscopic region, where 
the usual assumptions control the flow. An added complication is the fact that the position of the contact line is unknown, and therefore an additional relation is required between the contact angle and the slip velocity (see e.g. Haley \& Miksis 1991).

The above-mentioned ideas have also been used for surfactant-laden fluids. Cox (1986b) conducted a theoretical investigation, near the contact line, for the spreading liquid with an insoluble surfactant in the special case in which the surfactant does not transfer onto the solid. Cox recognized that, when surfactants are present, a singularity appears at the contact line just as in the pure fluid case and suggested that it could be removed by some suitable inner mechanism such as slip. However, Rame (2001) noted that when one considers the transfer of the surfactant from the fluid-air interface to the solid surface directly through the contact line by advection, further complications arise; the introduction of slip makes the fluid velocity relative to the contact line equal to zero, and therefore the surfactant cannot transfer by advection alone at the contact line. Clay \& Miksis (2004) also addressed the problem of transport of an insoluble surfactant at the contact line, albeit with a different mechanism, assuming a very simple model that permitted the surfactant to be adsorbed and desorbed at the contact line. Their results suggest that when the liquid-air interface is losing the surfactant to the substrate, through adsorption at the contact line, the droplet spreads faster, whereas when it is gaining the surfactant from the substrate the spreading becomes slower. This is a clear indication that the adsorption of the surfactant at the substrate plays an important role in the spreading process. More recently, Chan \& Borhan (2005) studied the axisymmetric spreading of a liquid drop with an insoluble surfactant and no transfer at the contact line. They focused on the effect of an equilibrium contact angle that either is fixed or depends on the surfactant concentration at the contact line.

Yet another approach to relieving the contact line singularity involves the use of a 'precursor' layer ahead of the contact line. This method has been used in several problems, e.g. the study of thin-film flow down an incline (Kondic \& Diez 2001), thermally driven coating flows (Kataoka \& Troian 1997), the spreading of a pure liquid on a solid substrate or on another liquid (Schwartz \& Eley 1998; Craster \& Matar 2006) and the stability of moving contact lines for Newtonian and viscoelastic fluids (Spaid \& Homsy 1996). This method has also been used in surfactant-laden flows, e.g. surfactant-induced fingering phenomena and the onset of 'autophobing' in thin-film flows (Edmonstone, Craster \& Matar 2006; Craster \& Matar 2007) as well as surfactant-enhanced spreading on a solid surface (Beacham, Matar \& Craster 2009). As we have already seen, however, up until very recently, the modelling work on surfactant-enhanced spreading on solid surfaces has focused on the case of insoluble surfactants (although there are a number of studies of the spreading of soluble surfactants on thin liquid films; Jensen \& Grotberg 1993; Warner, Craster \& Matar 2004a; Edmonstone et al. 2006). Beacham et al. (2009) accounted for a soluble surfactant with a concentration that can be below and above the critical micelle concentration (CMC), permitting, in the latter case, the formation of micellar aggregates. Moreover, these authors have taken into consideration the adsorption and desorption of the surfactant at the substrate. The adsorption of the surfactant at the substrate has already been recognized as an important factor by experimentalists and modellers in the spreading process (see e.g. Rafai et al. 2002; Kumar et al. 2003; Clay \& Miksis 2004; Kim, Qin \& Fichthorn 2006) as well as in other problems (e.g. the autophobing effect; see Craster \& Matar 2007). Furthermore, since the majority of the experimental studies, mentioned above, have considered soluble surfactants with concentrations that can be well above the CMC, the work of Beacham et al. 


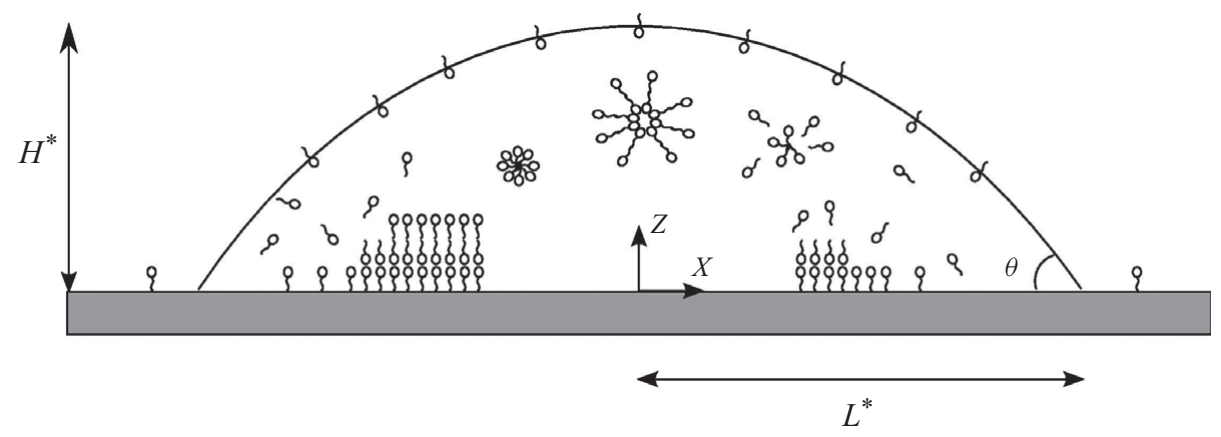

FIGURE 1. Schematic of a surfactant-laden drop spreading on a solid surface. The surfactant is present in the bulk as monomers or aggregates such as micelles or vesicles and at the fluid-air interface as monomers. If the substrate is hydrophobic the surfactant can be adsorbed as a mat or in other configurations.

(2009) represents an important step forward. Their results have been able to predict some of the experimental observations. Specifically, they were able to predict the nonmonotonicity in the spreading rates with increasing initial surfactant concentration. They have presented high spreading rates with power laws characterizing the droplet radius of up to $t^{2 / 3}$, and they have also predicted the formation of rims at the leading edge of the droplet as reported by Rafai et al. (2002) and Nikolov et al. (2002). We should note here, however, that although their model captures many of the essential elements of the problem at hand, it still lacks a significant component: a proper model of the contact line instead of a precursor layer.

The main objective of this paper is to model the surfactant-enhanced spreading of a drop on solid surfaces, taking into consideration the presence of a contact line explicitly. We consider both insoluble and soluble surfactants; in the latter case, the surfactant concentrations may exceed the CMC. Moreover, the surfactant in the bulk may be adsorbed/desorbed at the substrate, while the surfactant that resides at the interface can also be adsorbed/desorbed directly through the contact line. We use lubrication theory and a rapid-vertical-diffusion approximation to derive a coupled system of evolution equations for the drop profile and the surfactant concentration of the monomers and the micelles. The monomers exist in the bulk as well as at the liquid-air, liquid-solid and solid-air interfaces, whereas the micelles only exist in the bulk. The model accounts for Marangoni stresses, diffusion in the bulk and along the interfaces, micellar breakup and formation and sorption kinetics. The surface tensions are related to the surfactant concentrations through a nonlinear equation of state. The stress singularity at the contact line is alleviated by introducing slip in our model. Finally, we model the motion of the contact line through a constitutive relation that depends on the local surfactant concentration.

The rest of the paper is organized as follows. In $\S 2$, we describe the details of the evolution equations for the drop profile and the surfactant concentrations, while in $\S 3$ we present the numerical method used. Results are presented and discussed in $\S 4$, followed by concluding remarks in $\S 5$.

\section{Problem formulation}

We consider the dynamics of a drop of an incompressible Newtonian fluid with density $\rho^{*}$ and viscosity $\mu^{*}$, laden with surfactant, which has been deposited on a horizontal, rigid and impermeable solid substrate (see figure 1). The surface tensions 
of the liquid-air, liquid-solid and solid-air interfaces are $\sigma_{l}^{*}, \sigma_{l s}^{*}$ and $\sigma_{s}^{*}$, respectively. We assume that initially the drop has a maximal thickness $H^{*}$ and a half-width $L^{*}$. In the present work, we consider the drop to be very thin, and therefore $L^{*}$ is assumed to greatly exceed $H^{*}$ so that the drop aspect ratio $\varepsilon=H^{*} / L^{*}$ is assumed to be very small. The latter assumption permits the use of lubrication theory, which will be employed below to derive a set of evolution equations that govern the spreading process. The superscript $*$ indicates that the corresponding variable is dimensional.

\subsection{Hydrodynamics}

We use a Cartesian coordinate system $\left(x^{*}, z^{*}\right)$ to model the dynamics, and the velocity field is $\boldsymbol{u}^{*}=\left(u^{*}, w^{*}\right)$, where $u^{*}$ and $w^{*}$ correspond to the horizontal and vertical components of the velocity field, respectively. The liquid-air interface is located at $z^{*}=h^{*}\left(x^{*}, t^{*}\right)$, whereas the liquid-solid and the solid-air interfaces are located at $z^{*}=0$.

The spreading dynamics are governed by momentum and mass conservation equations, respectively given below:

$$
\begin{gathered}
\rho^{*}\left(\boldsymbol{u}_{t^{*}}^{*}+u^{*} \cdot \nabla \boldsymbol{u}^{*}\right)+\nabla p^{*}-\mu^{*} \nabla^{2} \boldsymbol{u}^{*}=0, \\
\nabla \cdot \boldsymbol{u}^{*}=0,
\end{gathered}
$$

where $\boldsymbol{u}^{*}$ and $p^{*}$ are the velocity vector and the pressure, respectively, while $\nabla$ denotes the gradient operator; the effect of gravitational force is negligible, and therefore the corresponding term has been neglected. Unless stated otherwise, the subscripts denote partial differentiation with respect to $x^{*}, z^{*}$ and $t^{*}$, where $t^{*}$ denotes time.

Solutions of (2.1) and (2.2) are obtained subject to the following boundary conditions. Along the free surface, the velocity field should satisfy a local force balance between surface tension and viscous stresses in the liquid, setting the pressure in the surrounding gas to zero (datum pressure) without loss of generality. Taking the tangential and normal to the free surface components of this force balance we obtain

$$
\begin{gathered}
\boldsymbol{n} \cdot \boldsymbol{T}^{*} \cdot \boldsymbol{t}=\boldsymbol{t} \cdot \nabla_{s} \sigma_{l}^{*}, \\
\boldsymbol{n} \cdot \boldsymbol{T}^{*} \cdot \boldsymbol{n}=2 \kappa^{*} \sigma_{l}^{*},
\end{gathered}
$$

where $\boldsymbol{n}=\left(-h_{x^{*}}^{*}, 1\right) /\left(1+h_{x^{*}}^{*}\right)^{1 / 2}$ and $\boldsymbol{t}=\left(1, h_{x^{*}}^{*}\right) /\left(1+h_{x^{*}}^{* 2}\right)^{1 / 2}$ denote the outward unit normal and unit tangential vectors on the interface, respectively; $\nabla_{s}$ is the surface gradient operator and $\boldsymbol{T}^{*}$ is the total stress tensor,

$$
\boldsymbol{T}^{*}=-p^{*} \boldsymbol{I}+\mu^{*}\left(\nabla \boldsymbol{u}^{*}+\left(\nabla \boldsymbol{u}^{*}\right)^{T}\right)
$$

where $\boldsymbol{I}$ is the identity tensor and $2 \kappa^{*}$ is the mean curvature of the free surface, defined as

$$
2 \kappa^{*}=-\nabla_{s} \cdot \boldsymbol{n}, \quad \nabla_{s}=(\boldsymbol{I}-\boldsymbol{n n}) \cdot \nabla .
$$

In addition, along the moving interface we impose the kinematic boundary condition,

$$
h_{t^{*}}^{*}+u^{*} h_{x^{*}}^{*}=w^{*} \quad \text { on } z^{*}=h^{*}\left(x^{*}, t^{*}\right)
$$

At the liquid-solid interface, two boundary conditions are imposed. In the vertical direction, we have the usual no-penetration condition:

$$
w^{*}=0 \text {. }
$$

In the horizontal direction, the usual no-slip condition is replaced by the slip condition of Navier (1823) to avoid the stress singularity, which would otherwise 
arise at the moving contact line. The slip model has the following form:

$$
u^{*}=\beta^{*} u_{z^{*}}^{*},
$$

where $\beta^{*}$ is a slip length.

\subsection{Surfactant transport and chemical kinetics}

We consider the spreading of a drop laden with either an insoluble or a soluble surfactant; the surfactant can also be adsorbed at the substrate. In the insoluble case, we assume that the surfactant exists only as a monomer at the liquid-air interface and at the solid surface, with concentrations $c_{a}^{*}$ and $c_{s}^{*}$, respectively. When we consider the case of a soluble surfactant we must take into consideration that once the monomer concentration is such that the bulk concentration of monomers is above the $\mathrm{CMC}, c_{c m c}^{*}$, then it is energetically favourable for the monomers to form micelles. We assume that below $c_{c m c}^{*}$ the surfactant exists in the form of monomers within the bulk with concentration $c^{*}$, whereas beyond $c_{c m c}^{*}$, micellar aggregates are formed with concentration $m^{*}$. The various surfactant species interact according to the following kinetic laws. First, at the liquid-air interface the transfer of surface monomers, $c_{a}^{*}$, into the bulk phase, $c^{*}$, creates space at the interface, or conversely, monomers from the bulk occupy space at the interface:

$$
S_{a}+c^{*} \underset{k_{2}^{*}}{\stackrel{k_{1}^{*}}{\rightleftharpoons}} c_{a}^{*} \text {. }
$$

Similarly, we model the adsorption of the surfactant at the substrate:

$$
S_{s}+c^{*} \underset{k_{4}^{*}}{\stackrel{k_{3}^{*}}{\rightleftharpoons}} c_{s}^{*},
$$

where $S_{i}(i=a, s)$ denotes the fraction of the total space created by the desorption of the monomers with concentration $c_{i}^{*}(i=a, s)$. We note here that our model assumes that there is no direct adsorption of the micelle aggregates at the interfaces: the micelles must disassociate first into monomers before being adsorbed at the interface. The micelles and the bulk monomers are related via

$$
N c^{*} \underset{k_{6}^{*}}{\stackrel{k_{5}^{*}}{\rightleftharpoons}} m^{*},
$$

which represents the creation of a micelle from $N$ bulk monomers or, conversely, the breakup of a micelle into $N$ bulk monomers. We have assumed here that there is a strongly preferred micelle size $N$, which is indeed often the case (Hunter 1991). The chemical kinetics are simple enough that modelling can proceed but still contain the essential physics that allows the model to capture realistic processes. The model can be adjusted to allow for more complicated behaviour, for, say, multiple micelle sizes, at the expense of further evolution equations; indeed the model is not restricted to micelles and could represent any aggregate of $N$ monomers in the bulk

A key detail is the behaviour of the chemical exactly at the contact line; monomers at the liquid-air interface could be adsorbed directly to the substrate or vice versa; that is, substrate monomers could be desorbed to the liquid-air interface through the contact line. This is modelled using the following 'reaction':

$$
S_{a}+c_{s}^{*} \underset{k_{8}^{*}}{\stackrel{k_{7}^{*}}{\rightleftharpoons}} S_{s}+c_{a}^{*},
$$


where the adsorption of surface monomers, $c_{a}^{*}$, occupies space at the substrate, $S_{s}$, creates space, $S_{a}$, at the interface and, conversely, as monomers, $c_{s}^{*}$, are desorbed from the substrate and adsorbed at the liquid-air interface. Note that each 'reaction' used for this model is characterized by a rate constant $k_{i}^{*}$, with $i=1,2, \ldots, 8$. We use these kinetic laws to generate the following fluxes that determine how the surfactant transfers between the different phases:

$$
\begin{gathered}
J_{b a}^{*}=-D_{c}^{*}\left[\boldsymbol{n} \cdot \nabla c^{*}\right]_{z^{*}=h^{*}}=\left.k_{1}^{*} c^{*}\right|_{z^{*}=h^{*}}\left(1-\frac{c_{a}^{*}}{c_{a \infty}^{*}}\right)-k_{2}^{*} c_{a}^{*}, \\
J_{b s}^{*}=-D_{c}^{*}\left[\boldsymbol{n} \cdot \nabla c^{*}\right]_{z^{*}=0}=\left.k_{3}^{*} c^{*}\right|_{z^{*}=0}\left(1-\frac{c_{s}^{*}}{c_{s \infty}^{*}}\right)-k_{4}^{*} c_{s}^{*}, \\
J_{b m}^{*}=k_{5}^{*} c^{* N}-k_{6}^{*} m^{*}, \\
J_{a s}^{*}=\left[k_{7}^{*} c_{s}^{*}\left(1-\frac{c_{a}^{*}}{c_{a \infty}^{*}}\right)-k_{8}^{*} c_{a}^{*}\left(1-\frac{c_{s}^{*}}{c_{s \infty}^{*}}\right)\right]_{x^{*}=x_{c}^{*}},
\end{gathered}
$$

where $x_{c}^{*}$ denotes the position of the contact line at time $t^{*}$. Here, $c_{i \infty}^{*}(i=a, s)$ represent the surfactant concentration at the liquid-air interface and at the substrate, respectively, at maximum packing. The nonlinear terms in (2.14), (2.15) and (2.17) imply that when $c_{i}^{*} \rightarrow c_{i \infty}^{*}(i=a, s)$, that is, when the liquid-air interface or the substrate becomes fully packed with monomers, no further surfactant is adsorbed. The nonlinear term comes from taking into consideration the available space $S_{i}$ $(i=a, s)$ at the substrate or interface, indicating that there is a limitation on the amount of monomers that can be adsorbed at both surfaces. At equilibrium, this is the Langmuir adsorption isotherm. This set of laws allows the surfactant to move from monomer to micelle and from bulk to either surface and also allows the surfactant to transfer through the contact line.

The behaviour of the various surfactant species is modelled by the following advection-diffusion equations:

$$
\begin{gathered}
c_{a_{t}^{*}}^{*}+\nabla_{s} \cdot\left(\boldsymbol{u}_{s}^{*} c_{a}^{*}\right)+c_{a}^{*}\left(\nabla_{s} \cdot \boldsymbol{n}\right)\left(\boldsymbol{u}^{*} \cdot \boldsymbol{n}\right)=D_{c a}^{*} \nabla_{s}^{2} c_{a}^{*}+J_{b a}^{*}, \\
c_{t^{*}}^{*}+\boldsymbol{u}^{*} \cdot \nabla c^{*}=D_{c}^{*} \nabla^{2} c^{*}-N J_{b m}^{*}, \\
m_{t^{*}}^{*}+\boldsymbol{u}^{*} \cdot \nabla m^{*}=D_{m}^{*} \nabla^{2} m^{*}+J_{b m}^{*}, \\
c_{s_{t}^{*}}^{*}=D_{c s}^{*} \nabla^{2} c_{s}^{*}+J_{b s}^{*},
\end{gathered}
$$

where $\boldsymbol{u}_{s}^{*}$ is the interfacial velocity defined as $\boldsymbol{u}_{s}^{*}=(\boldsymbol{I}-\boldsymbol{n n}) \boldsymbol{u}^{*}$ and $D_{i}^{*}(i=c a, c, m, c s)$ denotes the diffusion coefficients of the monomers at the liquid-air interface, of the monomers in the bulk, of the micelles and of the monomers at the substrate, respectively.

To complete the description, a constitutive equation that describes the dependence of the interfacial tensions on the surfactant concentrations is required. To this end, 
we use the Sheludko equation of state (Sheludko 1967; Gaver \& Grotberg 1990):

$$
\begin{gathered}
\sigma_{l}^{*}=\frac{\sigma_{l o}^{*}}{\left(1+\frac{c_{a}^{*}}{c_{a \infty}^{*}}\left[\left(\frac{\sigma_{l o}^{*}}{\sigma_{l m}^{*}}\right)^{1 / 3}-1\right]\right)^{3}}, \\
\sigma_{i}^{*}=\frac{\sigma_{i o}^{*}}{\left(1+\frac{c_{s}^{*}}{c_{s \infty}^{*}}\left[\left(\frac{\sigma_{i o}^{*}}{\sigma_{i m}^{*}}\right)^{1 / 3}-1\right]\right)^{3}}, \quad \text { for } i=l s, s,
\end{gathered}
$$

where $\sigma_{i o}^{*}$ and $\sigma_{i m}^{*}(i=l, l s, s)$ are the surface tension of a surfactant-free fluid and that of maximal surfactant concentration, respectively. This model is nonlinear and asymptotes to a minimal surface tension $\sigma_{i m}^{*}$ at high concentrations of the adsorbed surfactant, which makes it appropriate for use at high surfactant concentrations.

The total mass of the surfactant deposited per unit length, $M^{*}$, is a conserved quantity, given by

$$
\int_{0}^{x_{c}^{*}} \int_{0}^{h^{*}}\left(c^{*}+N m^{*}\right) \mathrm{d} z^{*} \mathrm{~d} x^{*}+\int_{0}^{x_{c}^{*}} c_{a}^{*} \mathrm{~d} x^{*}+\int_{0}^{\infty} c_{s}^{*} \mathrm{~d} x^{*}=M^{*},
$$

where we have used the symmetry of the problem to just consider $x>0$. Note that in the present model the surfactant monomers are permitted to diffuse on the substrate even beyond the contact line, and that is why the upper limit of the integral in the third term on the left-hand side of (2.24) tends to infinity.

\subsection{Scaling}

The governing equations and boundary conditions are made dimensionless, using the following scalings:

$$
\left.\begin{array}{l}
\left(x^{*}, z^{*}, h^{*}\right)=\left(L^{*} x, H^{*} z, H^{*} h\right), \quad t^{*}=\frac{L^{*}}{U^{*}} t, \quad\left(u^{*}, w^{*}\right)=\left(U^{*} u, \frac{U^{*} H^{*}}{L^{*}} w\right), \\
p^{*}=\frac{\mu^{*} U^{*} L^{*}}{H^{* 2}} p, \quad\left(c_{a}^{*}, c^{*}, m^{*}, c_{s}^{*}\right)=\left(c_{a \infty}^{*} c_{a}, c_{c m c}^{*} c, \frac{c_{c m c}^{*}}{N} m, c_{s \infty}^{*} c_{s}\right), \\
\left(J_{b a}^{*}, J_{b s}^{*}, J_{b m}^{*}, J_{a s}^{*}\right)=\left(\frac{U^{*} c_{a \infty}^{*}}{L^{*}} J_{b a}, \frac{U^{*} c_{s \infty}^{*}}{L_{b s}^{*}}, \frac{U^{*} c_{c m c}^{*}}{L^{*}} J_{b m}, U^{*} c_{a \infty}^{*} J_{a s}\right), \\
M^{*}=H^{*} L^{*} c_{c m c}^{*} M, \quad \sigma_{i}^{*}=\sigma_{i m}^{*}+\left(\sigma_{i o}^{*}-\sigma_{i m}^{*}\right) \sigma_{i} \quad(i=l, l s, s),
\end{array}\right\}
$$

where $U^{*}=\left(\sigma_{l o}^{*}-\sigma_{l m}^{*}\right) H^{*} / \mu^{*} L^{*}$ is a characteristic Marangoni velocity; $\sigma_{i o}^{*}$ and $\sigma_{i m}^{*}$ are the surface tension of zero and maximum surfactant concentration, respectively; and $c_{c m c}^{*}=\left(k_{6}^{*} / N k_{5}^{*}\right)^{1 /(N-1)}$.

Substitution of these scalings into the momentum and mass conservation governing equations and boundary conditions, using the lubrication approximation $\left(\varepsilon=H^{*} / L^{*} \ll 1\right)$, yields

$$
\begin{gathered}
p_{x}=u_{z z}, \quad p_{z}=0, \\
u_{x}+w_{z}=0, \\
p=-\varepsilon^{2} h_{x x}\left(\sigma_{l}+\frac{1}{\Sigma_{l}}\right), \quad u_{z}=\sigma_{l_{x}} \quad \text { at } z=h, \\
h_{t}+u h_{x}=w \quad \text { at } z=h, \\
u=\beta u_{z}, \quad w=0 \quad \text { at } z=0,
\end{gathered}
$$


where $\Sigma_{i}=\left(\sigma_{i o}^{*}-\sigma_{i m}^{*}\right) / \sigma_{i m}^{*}(i=l, s, l s)$ and $\beta$ is the dimensionless slip length, defined as $\beta=\beta^{*} / H^{*}$. It should be noted here that the lubrication approximation assumes small slopes, and therefore this model does not formally capture droplet spreading with high contact angles.

From the kinematic boundary condition and continuity ((2.29) and (2.27), respectively), we obtain

$$
h_{t}+(\bar{u} h)_{x}=0
$$

where $\bar{u}=(1 / h) \int_{0}^{h} u \mathrm{~d} z$. By solving (2.26), (2.28) and (2.30), we can derive the following expressions for the horizontal component of the velocity and pressure, respectively:

$$
\begin{gathered}
u=\frac{p_{x}}{2} z^{2}+\left(\sigma_{l_{x}}-p_{x} h\right) z+\beta\left(\sigma_{l x}-p_{x} h\right), \\
p=-\varepsilon^{2} h_{x x}\left(\sigma_{l}+\frac{1}{\Sigma_{l}}\right) .
\end{gathered}
$$

Using (2.32), we can also derive an expression for $\bar{u}$,

$$
\bar{u}=-\left(\frac{h^{2}}{3}+\beta h\right) p_{x}+\sigma_{l_{x}}\left(\beta+\frac{h}{2}\right),
$$

which results in the following evolution equation for $h$ :

$$
h_{t}=\left[\left(\frac{h^{3}}{3}+\beta h^{2}\right) p_{x}-\sigma_{l_{x}}\left(\beta h+\frac{h^{2}}{2}\right)\right]_{x} .
$$

The scaled surfactant transport equations become

$$
\begin{gathered}
c_{a_{t}}+\left(u_{s} c_{a}\right)_{x}=\frac{1}{P e_{c a}} c_{a_{x x}}+J_{b a}, \\
c_{t}+u c_{x}+w c_{x}=\frac{1}{P e_{c}}\left(c_{x x}+\frac{c_{z z}}{\varepsilon^{2}}\right)-J_{b m}, \\
m_{t}+u m_{x}+w m_{x}=\frac{1}{P e_{m}}\left(m_{x x}+\frac{m_{z z}}{\varepsilon^{2}}\right)+J_{b m}, \\
c_{s_{t}}=\frac{1}{P e_{c s}} c_{s_{x x}}+J_{b s},
\end{gathered}
$$

where $u_{s}=-\left(p_{x} / 2\right) h^{2}+\sigma_{l_{x}} h+\beta\left(\sigma_{l_{x}}-p_{x} h\right)$ is the interfacial velocity $(z=h)$. The dimensionless groups $P e_{i}=U^{*} L^{*} / D_{i}^{*}(i=c a, c, m, c s)$ are Péclet numbers representing a ratio of convective to diffusive time scales for the monomers at the free surface, the monomers and the micelles in the bulk and the monomers at the substrate, respectively. The dimensionless fluxes $J_{i}(i=b a, b s, b m, a s)$ that appear in the above equations are expressed by

$$
\begin{gathered}
J_{b a}=k_{a}\left(\left.R_{a} c\right|_{z=h}\left(1-c_{a}\right)-c_{a}\right), \\
J_{b s}=k_{s}\left(\left.R_{s} c\right|_{z=0}\left(1-c_{s}\right)-c_{s}\right), \\
J_{b m}=k_{b}\left(c^{N}-m\right), \\
J_{a s}=k_{a s}\left[R_{a s} c_{s}\left(1-c_{a}\right)-c_{a}\left(1-c_{s}\right)\right]_{x=x c},
\end{gathered}
$$

where the dimensionless parameters $k_{i}(i=a, s, b, a s)$ and $R_{i}(i=a, s, a s)$ are given by

$$
\begin{gathered}
k_{a}=\frac{k_{2}^{*} L^{*}}{U^{*}}, \quad k_{s}=\frac{k_{4}^{*} L^{*}}{U^{*}}, \quad k_{b}=\frac{k_{6}^{*} L^{*}}{U^{*}}, \quad k_{a s}=\frac{k_{8}^{*}}{U^{*}}, \\
R_{a}=\frac{k_{1}^{*} c_{c m c}^{*}}{k_{2}^{*} c_{a \infty}^{*}}, \quad R_{s}=\frac{k_{3}^{*} c_{c m c}^{*}}{k_{4}^{*} c_{s \infty}^{*}}, \quad R_{a s}=\frac{k_{7}^{*} c_{s \infty}^{*}}{k_{8}^{*} c_{a \infty}^{*}} .
\end{gathered}
$$


The kinetic parameters $k_{i}(i=a, s, b, a s)$ control the sorption kinetics at the free surface, the substrate, the breakup and formation rate of micellar aggregates in the bulk and the sorption kinetics at the contact line, respectively. The parameter $R_{i}$ $(i=a, s, a s)$ is a measure of the affinity of the surfactant to the liquid-air interface and substrate. Small values of $R_{a}$ and $R_{s}$ signify the tendency of the surfactant to remain in the bulk in the form of micelles (Edmonstone et al. 2006). Similarly, when $R_{a s}$ is small the surfactant at the contact line has the tendency to remain at the liquid-air interface instead of being adsorbed at the substrate.

Finally, the dimensionless forms of the Sheludko equation of state for all the interfaces are given by

$$
\begin{gathered}
\sigma_{l}=\left(\frac{1+\Sigma_{l}}{\Sigma_{l}}\right)\left(1+c_{a}\left[\left(1+\Sigma_{l}\right)^{1 / 3}-1\right]\right)^{-3}-\frac{1}{\Sigma_{l}}, \\
\sigma_{i}=\left(\frac{1+\Sigma_{i}}{\Sigma_{i}}\right)\left(1+c_{s}\left[\left(1+\Sigma_{i}\right)^{1 / 3}-1\right]\right)^{-3}-\frac{1}{\Sigma_{i}}, \quad \text { for } i=l s, s .
\end{gathered}
$$

\subsection{Rapid vertical diffusion}

We assume vertical diffusion to be rapid and use an approach previously followed in the literature (Jensen \& Grotberg 1993). The rapid-diffusion assumption is equivalent to making the substitutions

$$
\begin{gathered}
c(x, z, t)=c_{0}(x, t)+\varepsilon^{2} P e_{c} c_{1}(x, z, t), \\
m(x, z, t)=m_{0}(x, t)+\varepsilon^{2} P e_{m} m_{1}(x, z, t)
\end{gathered}
$$

in (2.36)-(2.39) and then averaging in the vertical direction the monomer and micelle equations in the bulk, under the assumption that $\left(\bar{c}_{1}, \bar{m}_{1}\right)=(1 / h) \int_{0}^{h}\left(c_{1}, m_{1}\right) \mathrm{d} z=0$, taking the limit $\varepsilon^{2} P e_{i} \rightarrow 0(i=c, m)$. In addition, we employ the boundary conditions

$$
\begin{gathered}
J_{b a}=-\frac{1}{\beta_{a}}\left(-\frac{h_{x}}{P e_{c}} c_{0_{x}}+c_{1_{z}}\right)_{z=h}, \\
J_{b s}=\left.\frac{1}{\beta_{s}} c_{1_{z}}\right|_{z=h}
\end{gathered}
$$

in the $z$-direction for the monomers; these conditions are the dimensionless form of (2.14) and (2.15), as well as the ones for the micelles,

$$
\left.m_{1_{z}}\right|_{z=h}=\left.m_{1_{z}}\right|_{z=0}=0,
$$

since, as was mentioned above, we assume that there is no direct adsorption of micelles at the liquid-air interface or the substrate. The dimensionless parameters $\beta_{a}$ and $\beta_{s}$ provide a measure of surfactant solubility in the bulk fluid and are given by

$$
\beta_{a}=\frac{c_{a \infty}^{*}}{H^{*} c_{c m c}^{*}}, \quad \beta_{s}=\frac{c_{s \infty}^{*}}{H^{*} c_{c m c}^{*}} .
$$

After dropping the subscript 0 , we obtain the following equations:

$$
\begin{gathered}
c_{a_{t}}+\left(u_{s} c_{a}\right)_{x}=\frac{c_{a_{x x}}}{P e_{c a}}+J_{b a}, \\
c_{t}+\bar{u} c_{x}=\frac{\left(h c_{x}\right)_{x}}{h P e_{c}}-\frac{\beta_{a}}{h} J_{b a}-\frac{\beta_{s}}{h} J_{b s}-J_{b m},
\end{gathered}
$$




$$
\begin{gathered}
m_{t}+\bar{u} m_{x}=\frac{\left(h m_{x}\right)_{x}}{h P e_{m}}+J_{b m}, \\
c_{s_{t}}=\frac{c_{s_{x x}}}{P e_{c s}}+J_{b s} .
\end{gathered}
$$

Finally, the total dimensionless mass of surfactant is given by

$$
\int_{0}^{x_{c}} h(c+m) \mathrm{d} x+\beta_{a} \int_{0}^{x_{c}} c_{a} \mathrm{~d} x+\beta_{s} \int_{0}^{\infty} c_{s} \mathrm{~d} x=M .
$$

When we consider the case of an insoluble surfactant, the bulk concentrations of the monomers, $c$, and the micelles, $m$, as well as all the related fluxes are equal to zero. Thus, in this case the total mass of the surfactant is given by

$$
\int_{0}^{x_{c}} c_{a} \mathrm{~d} x+\beta_{a s} \int_{0}^{\infty} c_{s} \mathrm{~d} x=M^{\prime}
$$

where $\beta_{a s}=c_{s \infty}^{*} / c_{a \infty}^{*}$ and $M^{\prime}=M^{*} /\left(L^{*} c_{a \infty}^{*}\right)$.

\subsection{Contact line motion}

The contact line is a moving boundary, and therefore additional information is needed in order to determine its spatio-temporal evolution. Since the detailed physics very close to the contact line is not clear yet, we have decided to use an empirical constitutive equation which relates the fluid velocity at the contact line with the contact angle (Tanner 1979). In its dimensionless form, this relation is given by

$$
\frac{\mathrm{d} x_{c}}{\mathrm{~d} t}=k\left(\theta-\theta_{a}\right)^{n}
$$

where $\theta$ is the dynamic contact angle; $\theta_{a}$ is the equilibrium advancing contact angle; and $k=\varepsilon^{n} k^{*} / U^{*}$. The model has two empirical constants, the so-called mobility exponent $k^{*}$ and $n$ which usually takes values in the range $1 \leqslant n \leqslant 3$. This functional dependence has been used by several researchers in the past to model contact line motion (Haley \& Miksis 1991; Benintendi \& Smith 1999; Chan \& Borhan 2005). The power-law dependence has been verified experimentally for the spreading of uncontaminated fluids (e.g. see Ehrhard 1993).

It is expected that the presence of surfactants, apart from affecting the interfacial forces, will also have a significant effect on the forces at the contact line. We assume, however, that the same power-law dependence is valid in the presence of surfactants. It is also reasonable to assume that the variation of the surfactant concentration during the spreading process will have an effect on the equilibrium contact angle. At equilibrium, and in the general case in which surfactants are present, the balance between the horizontal interfacial forces at the contact point in dimensional form gives

$$
\sigma_{s}^{*}=\sigma_{l}^{*} \cos \theta_{a}^{*}+\sigma_{l s}^{*} .
$$

Similarly, for a clean fluid we get

$$
\sigma_{s o}^{*}=\sigma_{l o}^{*} \cos \theta_{a c}^{*}+\sigma_{l s o}^{*},
$$

where $\theta_{a c}^{*}$ is the corresponding equilibrium contact angle. We set $\theta_{i}^{*}=\varepsilon \theta_{i}(i=a, a c)$ and $\sigma_{i}^{*}=\sigma_{i m}^{*}+\left(\sigma_{i o}^{*}-\sigma_{i m}^{*}\right) \sigma_{i}(i=l, l s, s)$ and substitute (2.62) in (2.61) to get

$$
\cos \left(\varepsilon \theta_{a}\right)=\frac{\left(1+\Sigma_{l}\right) \sigma_{s} \cos \left(\varepsilon \theta_{a c}\right)+\left(\delta_{s}-\delta_{l s}\right)\left(1-\sigma_{s}\right)+\Sigma_{l s} \delta_{l s}\left(\sigma_{s}-\sigma_{l s}\right)}{\left(1+\Sigma_{l} \sigma_{l}\right)},
$$


where $\delta_{i}=\sigma_{i m}^{*} / \sigma_{l m}^{*}(i=l s, s)$. Using the fact that $\cos (\varepsilon \theta)=1-\varepsilon^{2} \theta^{2} / 2$, since $\varepsilon \ll 1$ in the lubrication limit, we can then derive the following relation:

$$
\theta_{a}^{2}=\frac{\left(1+\Sigma_{l}\right) \sigma_{s} \varepsilon^{2} \theta_{a c}^{2}+2\left[\left(1-\delta_{s}+\delta_{l s}\right)\left(1-\sigma_{s}\right)+\Sigma_{l}\left(\sigma_{l}-\sigma_{s}\right)+\Sigma_{l s} \delta_{l s}\left(\sigma_{l s}-\sigma_{s}\right)\right]}{\varepsilon^{2}\left(1+\Sigma_{l} \sigma_{l}\right)} .
$$

This expression for $\theta_{a}$ is used in (2.60) to model the local influence of surfactants on the contact line velocity. Moreover, when the right-hand side of (2.64) becomes negative, we simply assume that $\theta_{a}=0$.

\section{Numerical method}

\subsection{Finite-element method}

The discretization of the governing equations is performed using a finiteelement/Galerkin method, and we approximate all the variables through the use of quadratic Lagrangian basis functions $\phi^{i}$. Applying the divergence theorem, the weak form of the equations for the position of the liquid-air interface, $h$, and the surfactant monomers at the free surface become

$$
\begin{gathered}
\int_{0}^{x_{c}}\left(h_{t} \phi_{i}-\bar{u} h \phi_{i_{x}}\right) \mathrm{d} x+\left[\bar{u} h \phi_{i}\right]_{0}^{x_{c}}=0, \\
\int_{0}^{x_{c}}\left[\left(c_{a_{t}}-J_{b a}\right) \phi_{i}-\left(u_{s} c_{a}-\frac{c_{a_{x}}}{P e_{c a}}\right) \phi_{i_{x}}\right] \mathrm{d} x+\left[u_{s} c_{a}-\frac{c_{a_{x}}}{P e_{c a}}\right]_{0}^{x_{c}}=0 .
\end{gathered}
$$

The presence of $h$ in the denominator of several terms in (2.55) and (2.56) will cause significant numerical difficulties close to the contact line, since in that region $h \rightarrow 0$. To overcome this problem, we multiply through by $h$, and thus the corresponding weak forms, after applying the divergence theorem, become

$$
\begin{gathered}
\int_{0}^{x_{c}}\left[\left(h c_{t}+h \bar{u} c_{x}+\beta_{a} J_{b a}+\beta_{s} J_{b s}+h J_{b m}\right) \phi_{i}+h c_{x} \phi_{i_{x}}\right] \mathrm{d} x-\left[\frac{h c_{x}}{P e_{c}}\right]_{0}^{x_{c}}=0, \\
\int_{0}^{x_{c}}\left[\left(h m_{t}+h \bar{u} m_{x}-h J_{b m}\right) \phi_{i}+h m_{x} \phi_{i_{x}}\right] \mathrm{d} x-\left[\frac{h m_{x}}{P e_{m}}\right]_{0}^{x_{c}}=0 .
\end{gathered}
$$

The weak form of the equation for the surfactant that is adsorbed at the substrate, $c_{s}$, is as follows:

$$
\begin{aligned}
\int_{0}^{x_{c}^{-}}\left[\left(c_{s_{t}}-J_{b s}\right) \phi_{i}+\right. & \left.\frac{c_{s_{x}}}{P e_{c s}} \phi_{i_{x}}\right] \mathrm{d} x-\left[\frac{c_{s_{x}}}{P e_{c s}}\right]_{0}^{x_{c}^{-}} \\
& +\int_{x_{c}^{+}}^{\infty}\left[\left(c_{s_{t}}-J_{b s}\right) \phi_{i}+\frac{c_{s_{x}}}{P e_{c s}} \phi_{i_{x}}\right] \mathrm{d} x-\left[\frac{c_{s_{x}}}{P e_{c s}}\right]_{x_{c}^{+}}^{\infty}=0 .
\end{aligned}
$$

Note that the integral is split into two parts, one inside the drop and one outside it, for reasons that will be made clear below.

\subsection{Boundary conditions}

To solve the above set of equations we need to impose appropriate boundary equations in the $x$-direction which are applied by substituting the boundary terms in (3.1)-(3.5). At the plane of symmetry we apply symmetry conditions

$$
h_{x}=h_{x x x}=0, \quad c_{a_{x}}=c_{x}=m_{x}=c_{s_{x}}=0 \quad \text { at } x=0 .
$$


We expect that the concentration of the surfactant, which is adsorbed at the substrate, should not be affected by the presence of the drop very far from it; thus we impose

$$
c_{s_{x}}=0 \quad \text { at } x \rightarrow \infty .
$$

Although the boundary condition for $h$ is obvious at the contact line,

$$
h=0 \quad \text { at } x=x_{c},
$$

this is certainly not the case for $c$ and $m$. We have to keep in mind that both $c$ and $m$ are volume concentrations, while the volume of the fluid at $x=x_{c}$ reduces to a line, making them both singular. Fortunately, there is no need to find such a boundary condition for these variables, since the corresponding terms in (3.3) and (3.4) are multiplied by $h$ which is zero-valued at $x=x_{c}$ according to (3.8).

As mentioned earlier our model permits the transfer of surfactant monomers from the free surface to the substrate and, conversely, directly through the contact line. The associated flux for a soluble surfactant is given by

$$
\left.\frac{c_{s_{x}}}{P e_{c s}}\right|_{x=x_{c}^{+}}-\left.\frac{c_{s_{x}}}{P e_{c s}}\right|_{x=x_{c}^{-}}=\frac{\beta_{a}}{\beta_{s}} J_{a s},
$$

which, in the case of an insoluble surfactant, becomes

$$
\left.\frac{c_{s_{x}}}{P e_{c s}}\right|_{x=x_{c}^{+}}-\left.\frac{c_{s_{x}}}{P e_{c s}}\right|_{x=x_{c}^{-}}=\frac{1}{\beta_{a s}} J_{a s} .
$$

This relation is used as a boundary condition for $c_{s}$; the application of this boundary condition is the reason for splitting the integral in (3.5) into two parts.

To derive the appropriate boundary condition for $c_{a}$ at $x=x_{c}$, we use conservation of surfactant mass. Differentiating (2.58) with respect to $t$ yields

$$
\frac{\mathrm{d}}{\mathrm{d} t} \int_{0}^{x_{c}} h(c+m) \mathrm{d} x+\beta_{a} \frac{\mathrm{d}}{\mathrm{d} t} \int_{0}^{x_{c}} c_{a} \mathrm{~d} x+\beta_{s} \frac{\mathrm{d}}{\mathrm{d} t} \int_{0}^{\infty} c_{s} \mathrm{~d} x=0,
$$

since we know that $\mathrm{d} M / \mathrm{d} t=0$. We proceed to calculate every term of this equation. We start by integrating (2.54) which, after using Leibniz's rule, becomes

$$
\frac{\mathrm{d}}{\mathrm{d} t} \int_{0}^{x_{c}} c_{a} \mathrm{~d} x=\left.\frac{\mathrm{d} x_{c}}{\mathrm{~d} t} c_{a}\right|_{x=x_{c}}-\left[u_{s} c_{a}-\frac{c_{a_{x}}}{P e_{c a}}\right]_{x=x_{c}}+\int_{0}^{x_{c}} J_{b a} \mathrm{~d} x .
$$

Multiplying (2.55) and (2.56) with $h$ and integrating in $x$ we get

$$
\begin{gathered}
\frac{\mathrm{d}}{\mathrm{d} t} \int_{0}^{x_{c}} h c \mathrm{~d} x=-\int_{0}^{x_{c}}\left(\beta_{a} J_{b a}+\beta_{s} J_{b s}+h J_{b m}\right) \mathrm{d} x, \\
\frac{\mathrm{d}}{\mathrm{d} t} \int_{0}^{x_{c}} h m \mathrm{~d} x=\int_{0}^{x_{c}} h J_{b m} \mathrm{~d} x .
\end{gathered}
$$

Next, we integrate (2.58) and split the integral into two parts, one inside the fluid drop and one outside it, as in (3.5). After applying (3.6) and (3.7) the equation becomes

$$
\frac{\mathrm{d}}{\mathrm{d} t} \int_{0}^{\infty} c_{s} \mathrm{~d} x=\left.\frac{c_{s_{x}}}{P e_{c s}}\right|_{x=x_{c}^{-}}-\left.\frac{c_{s_{x}}}{P e_{c s}}\right|_{x=x_{c}^{+}}+\int_{0}^{x_{c}} J_{b s} \mathrm{~d} x
$$


Finally, we substitute (3.12)-(3.15) and (3.9) in (3.11), and we end up with the following boundary condition for $c_{a}$ :

$$
\left[u_{s} c_{a}-\frac{c_{a_{x}}}{P e_{c a}}\right]_{x=x_{c}}=\left.\frac{\mathrm{d} x_{c}}{\mathrm{~d} t} c_{a}\right|_{x=x_{c}}-J_{a s} .
$$

This boundary condition has been derived here for soluble surfactants, but it can be readily shown that it holds also for the case of insoluble ones. We should note that at the contact line $\left(x=x_{c}\right)$ the interfacial velocity $u_{s}$ which appears on the left-hand side of (3.16) is equal to the contact line velocity $\mathrm{d} x_{c} / \mathrm{d} t$.

\subsection{Initial conditions}

The initial condition used for the film thickness, the position of the contact line and the surfactant concentrations are given by

$$
\begin{aligned}
h(x, t=0) & =1-x^{2}, \\
x_{c}(t=0) & =1, \\
\left(c_{a}, c, m, c_{s}\right)(x, t=0) & =\left(c_{a o}, c_{o}, m_{o}, 0\right) .
\end{aligned}
$$

We assume that at $t=0$ the surfactant concentrations are in local equilibrium, and hence the fluxes $J_{b a}=J_{b s}=0$. Thus, we have

$$
c_{o}=m_{o}^{1 / N}, \quad c_{a o}=\frac{R_{a} m_{o}^{1 / N}}{1+R_{a} m_{o}^{1 / N}} .
$$

Substitution of (3.20) into (2.58) yields

$$
\frac{2}{3}\left(m_{o}^{1 / N}+m_{o}\right)+\frac{\beta_{a} R_{a} m_{o}^{1 / N}}{1+R_{a} m_{o}^{1 / N}}=M,
$$

which is solved numerically for a prescribed value of $M$.

If $M<1$, then the surfactant concentration is below the CMC, and consequently no micelles are present. In that case, we set $m_{o}=0$, and the rest of the concentrations are found solving

$$
\frac{2}{3} c_{o}+\frac{\beta_{a} R_{a} c_{o}}{1+R_{a} c_{o}}=M, \quad c_{a o}=\frac{R_{a} c_{o}}{1+R_{a} c_{o}} .
$$

Finally, in the case of an insoluble surfactant there are no surfactant monomers in the bulk; therefore $c_{o}=0$ and the initial condition for $c_{a o}$ is given by

$$
c_{a o}=M^{\prime} .
$$

\subsection{Mapping}

The physical domain consists of two parts, one inside the fluid drop, which is finite, and one outside it, which is infinite. During the spreading process the contact line moves, and therefore the physical domain changes with time. In order to map the transient physical domain, $(x, t)$, onto a computational domain fixed in time, $(\eta, \tau)$, we use the following set of algebraic equations:

$$
\eta=\left\{\begin{array}{ll}
\frac{x}{x_{c}}, & 0 \leqslant x \leqslant x_{c}, \\
2-\frac{x_{c}}{x}, & x \geqslant x_{c},
\end{array} \text { and } \tau=t .\right.
$$


The interior of the drop is mapped to $0 \leqslant \eta \leqslant 1$, while the infinite physical domain outside the drop is mapped onto a finite computational one, $1 \leqslant \eta \leqslant 2$. The derivatives that arise in the evolution equations also have to be rewritten in terms of the new variables

$$
\begin{gathered}
\partial_{t}=\partial_{\tau}-\frac{\eta}{x_{c}} \frac{\mathrm{d} x_{c}}{\mathrm{~d} t} \partial_{\eta}, \quad \partial_{x}=\frac{1}{x_{c}} \partial_{\eta} \quad \text { for } 0 \leqslant \eta \leqslant 1, \\
\partial_{t}=\partial_{\tau}-\frac{2-\eta}{x_{c}} \frac{\mathrm{d} x_{c}}{\mathrm{~d} t} \partial_{\eta}, \quad \partial_{x}=\frac{(2-\eta)^{2}}{x_{c}} \partial_{\eta} \quad \text { for } 1 \leqslant \eta \leqslant 2
\end{gathered}
$$

and are used to replace the corresponding terms in the weak form of the governing equations presented above as well as in the corresponding boundary conditions. Both computational domains are discretized using 300 elements in all the computations presented in this paper; numerical checks showed that increasing the number of elements further led to negligible changes. In all the simulations presented below, the fluid and the surfactant mass conservation are satisfied within $1 \%$ and $0.01 \%$, respectively.

The resulting set of discrete equations is integrated in time with the implicit Euler method. An automatically adjusted time step is used for that purpose, which ensures convergence and optimizes code performance. The initial time step for all the simulations was $\Delta t=10^{-7}$. The final set of algebraic equations is nonlinear, and they are solved in each time step, using the Newton-Raphson method. The iterations of the Newton-Raphson method are terminated using $10^{-9}$ as tolerance for the absolute error of the residual vector. The code was written in Fortran 90 and was run on a personal computer with Intel Core 2 Duo E8400 at $3 \mathrm{GHz}$. Each run typically required 3-6 h to complete.

\section{Results and discussion}

The spreading of a surfactant-laden liquid drop is a parametrically rich problem. We begin our study by examining the case of an insoluble surfactant in $\S 4.1$, while in $\S 4.2$ we present simulations for a soluble surfactant the concentration of which may exceed the CMC. Numerical solutions were obtained over a wide range of parameter values. The 'base' case, however, has broadly typical values of $\varepsilon^{2}=0.005, \Sigma_{s}=0.2$, $\Sigma_{l}=\Sigma_{l s}=2, \delta_{s}=5, \delta_{l s}=1, \beta=10^{-5}, k=0.01, n=3, \theta_{a c}=1$. This set of parameters corresponds to the spreading of slender drops in the presence of an insoluble or a soluble surfactant.

\subsection{Insoluble surfactant}

\subsubsection{No adsorption at the contact line}

To set the stage we begin with the simplest configuration, i.e. the spreading of a drop with an insoluble surfactant and no adsorption at the contact line. The typical drop evolution and the associated surfactant monolayer concentration at the interface are presented in figure 2. As the initial condition we use (3.17), so the drop has a parabolic profile with contact angle higher than its equilibrium value; the drop naturally spreads, and as a result, the contact line moves along the solid surface, while the maximum height of the drop decreases with time. The contact angle decreases as the drop spreads, tending to its equilibrium value, and consequently, the spreading rate decelerates; spreading stops when the equilibrium contact angle is reached. The concentration of surfactant monolayer decreases significantly with time, as there is considerable dilation of the interface. 

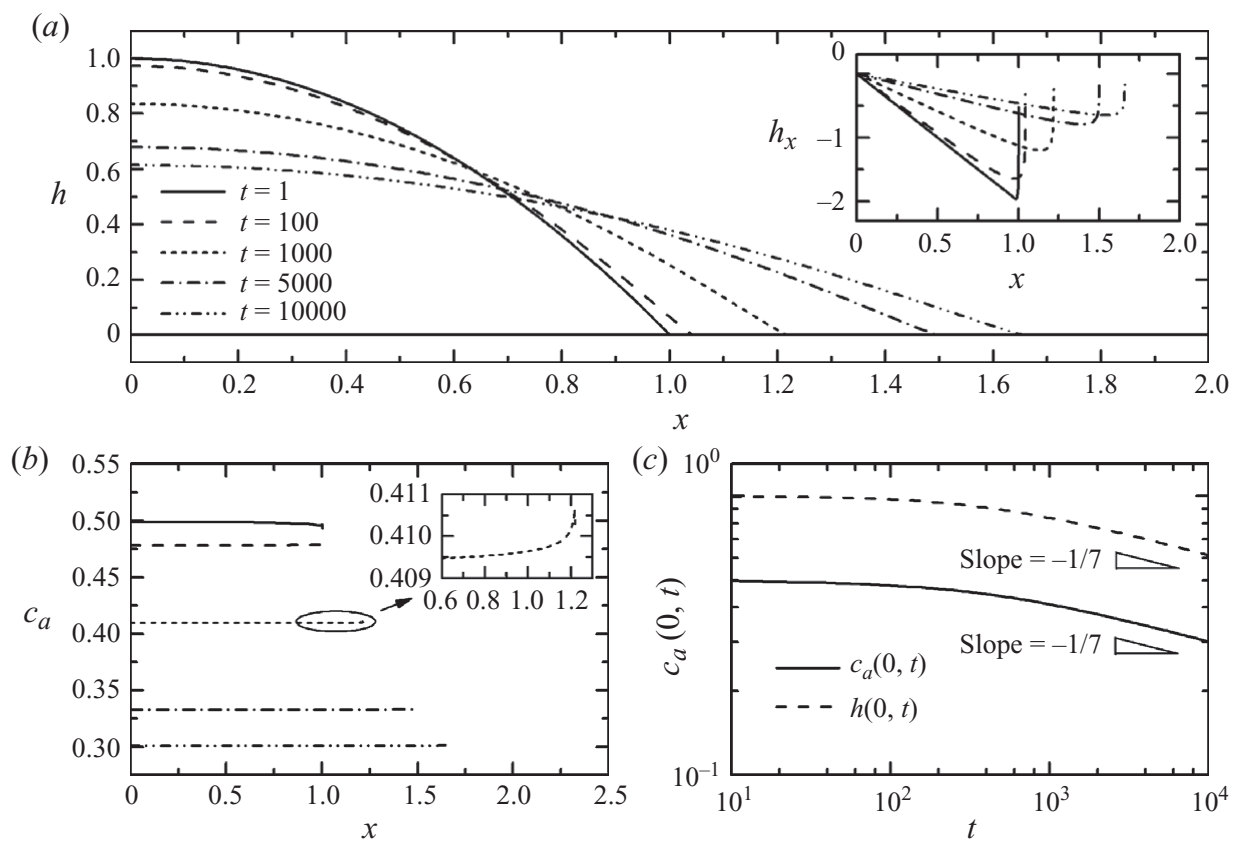

FIgURE 2. Time evolution of $(a)$ the drop profile and $(b)$ the surfactant concentration for an insoluble surfactant with $\left(M, k_{a s}, P e_{c a}\right)=\left(0.5,0,10^{4}\right) .(c) h(0, t)$ and $c_{a}(0, t)$.

The concentration is almost spatially uniform along the interface, although the Péclet number is large, and therefore advection of the surfactant is the dominant mechanism for its transport. This concentration profile arises because the dilation of the interface near the contact line compensates for the additional surfactant that is transferred there by advection. Nevertheless, there are small variations in the concentration profile, which are shown in more detail in the inset of figure $2(b)$. We can see that there is a monotonic increase of the surfactant concentration towards the contact point as the surfactant accumulates in the region around the contact line. The accumulation of the surfactant induces negative surface tension gradients, which result in a Marangoni flow that opposes spreading. Close to the contact line there is also smaller resistance to the deformations of the interface because of the lower surface tension. Thus, the Marangoni flow, which drives fluid from the contact line towards the centre of the drop, affects the shape of the interface by changing its curvature close to the contact line, forcing it to turn from convex to concave, decreasing drastically the contact angle tending to its equilibrium value. This is shown clearly in figure $2(a)$ in which we have plotted the derivative of $h$; the position of its minimum value corresponds to the stationary point. This behaviour was also observed by Chan \& Borhan (2005). Figure 2(c) presents the evolution of the drop thickness and surfactant concentration at the plane of symmetry. We can see that at late times both $h(0, t)$ and $c_{a}(0, t)$ scale with $t^{-1 / 7}$, and the leading edge $x_{c}$ scales as $t^{1 / 7}$. These scalings are the same as those for a surfactant-free droplet spreading with the same contact line model (Ehrhard \& Davis 1991); the scaling for the surfactant follows from surfactant mass conservation. It is worth a brief comparison with the spreading of a surfactant-laden droplet over a pre-wetted substrate (Warner, Craster \& Matar 2004b; Edmonstone et al. 2006; Jensen \& Naire 2006) for which the droplet spreads 

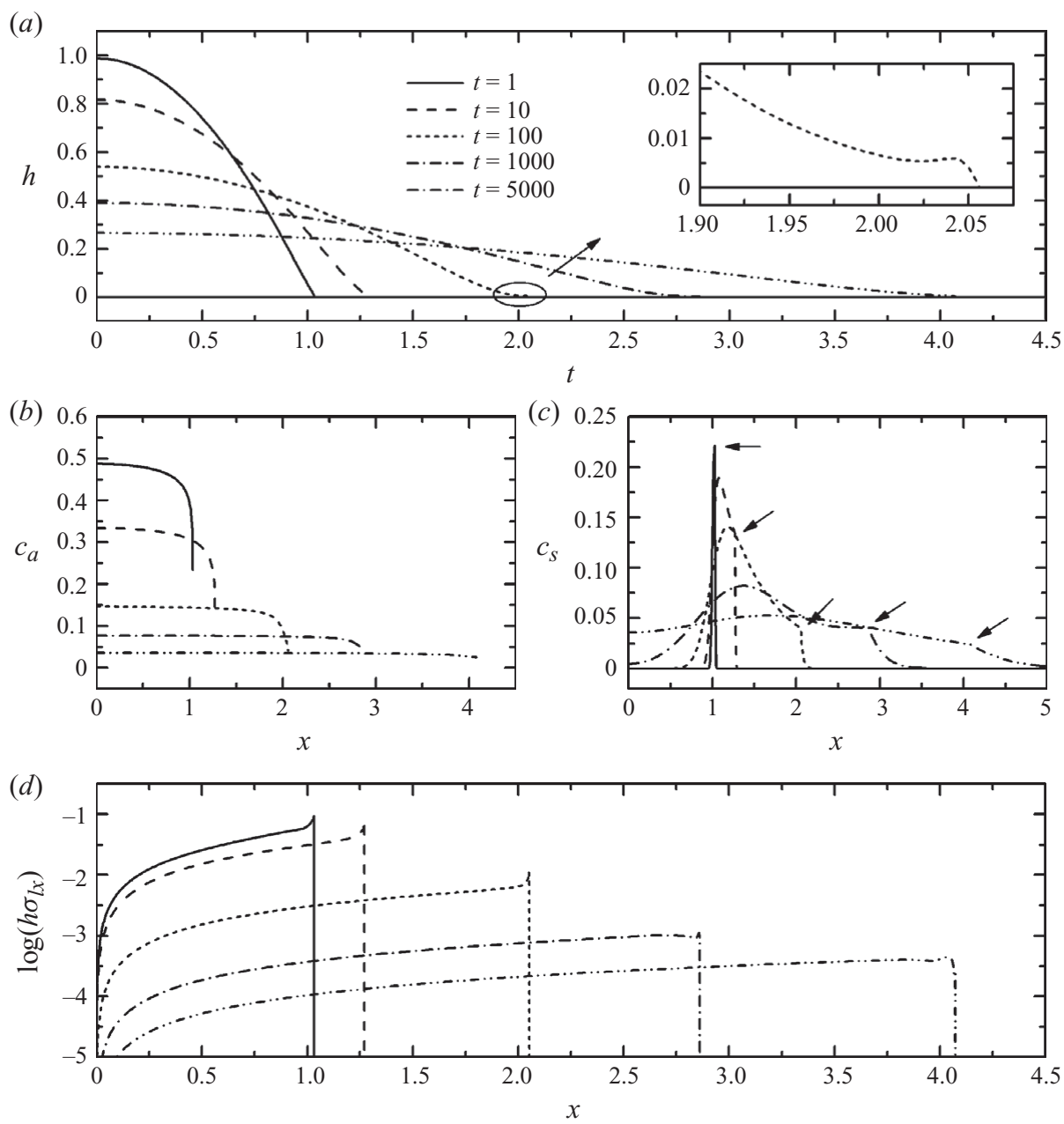

FiguRE 3. Time evolution of $(a)$ the drop profile, the surfactant concentrations at the $(b)$ liquid-air and $(c)$ liquid-solid interfaces and $(d)$ the Marangoni stresses at the liquid-air interface for an insoluble surfactant with $\left(M, \beta_{a s}, k_{a s}, P e_{c a}, P e_{c s}\right)=\left(0.5,2,1,10^{4}, 10^{4}\right)$. The arrows in $(c)$ indicate the position of the contact line.

with the same scalings; the concentration remains broadly spatially constant in the droplet, but it decreases there as $t^{-2 / 7}$, as there is a flux of surfactant out through the narrow gap at the droplet edge onto the pre-wetted film, driving the evolution of an outer rim; this feature is absent here.

\subsubsection{Adsorption/desorption at the substrate through the contact line}

Next, we discuss the situation in which an insoluble surfactant can be both adsorbed and desorbed at the substrate. Figure 3 shows the evolution of the drop profiles as well as surfactant concentration profiles at the liquid-air and liquid-solid interfaces. As the surfactant is insoluble it can only be adsorbed and desorbed at the substrate directly through the contact line at which the substrate and the liquid-air interface meet. Once the surfactant is adsorbed at the solid surface it can diffuse in both directions, i.e. towards the centre of the drop as well as ahead of the contact line. Initially the substrate is considered to be clean, and therefore the concentration of 
the surfactant monolayer there is zero. As soon as the drop comes into contact with the substrate, the surfactant which is present at the liquid-air interface is adsorbed at the solid surface through the contact line. Consequently, as shown in figure 3(c), at very early times the surfactant concentration on the substrate is zero everywhere except for the contact line and a small region around it, because of surface diffusion, which, for $P e_{c s}=10^{4}$, is rather weak. The adsorption of the surfactant at the solid wall results in an abrupt decrease of the surfactant concentration at the liquid-air interface very close to the contact line (see figure $3 b$ ). This decrease becomes less prominent with increasing time, since less surfactant is adsorbed at the substrate. This happens mainly for two reasons. The first is that the surfactant concentration $c_{a}$ decreases considerably with time because of the dilation of the interface. The second is that at large times, enough surfactant has already diffused ahead of the contact line, and consequently the rate of adsorption decreases significantly.

The rapid decrease of $c_{a}$ near the contact line leads to locally large positive surface tension gradients and consequently to a Marangoni flow that promotes spreading. Figure $3(d)$ shows the evolution of the Marangoni stresses which increase strongly as we approach the contact line. This is in contrast to the case of no adsorption, where the accumulation of surfactant close to the contact point actually induces a Marangoni flow that opposes spreading. Indeed, comparing figures $2(a)$ and $3(a)$, spreading is much faster when the surfactant is allowed to be adsorbed at the substrate. This is slightly counter-intuitive, as one naturally feels that adsorption will remove the surfactant and thereby reduce any driving effect created by it; however, the important point is that it is not the surfactant concentration that drives flow but its gradient. As noted by Kim et al. (2006), who performed molecular dynamics simulations on the spreading of nano-droplets laden with an insoluble surfactant, when the adsorption of the surfactant at the liquid-solid interface becomes significant, the concentration of the surfactant monomers at the liquid-air interface in the vicinity of the contact line decreases significantly, which results in enhanced spreading due to the induced Marangoni stresses; this is in agreement with our findings. The effect of surfactant adsorption is also significant on the drop shape near the contact line at which the local Marangoni stresses, and the flow that they drive, create a small ridge (see the inset of figure $3 a$ ). In addition, the high surface tension near the contact line provides a larger resistance to the deformation of the interface. As a result, the shape of the interface very close to the contact line remains convex and does not become concave, in contrast with the zero-adsorption case (see figure $2 a$ and the relevant discussion). The convex shape leads to values of the contact angle that remain large for longer times (see also figure $4 c, d$ ), and therefore the drop spreads faster; this is a direct consequence of the empirical constitutive equation (2.60) used to model the motion of the contact line.

\subsubsection{Effect of initial surfactant concentration and sorption kinetics}

To explore the effect of different surfactants on the spreading rates we choose various values of $M$ (the initial mass of surfactant emplaced) and $k_{a s}$ (the parameter controlling the kinetics of adsorption at the contact line) while keeping $\beta_{a s}$ (a solubility parameter, $\beta_{a s}=c_{s \infty}^{*} / c_{a \infty}^{*}$, measuring the affinity of surfactant for the substrate versus that of the interface) fixed with $\beta_{a s}=2$, as presented in figure 4(a). First, we examine the effect of varying the kinetic parameter $k_{a s}$, keeping the initial surfactant mass constant with $M=0.5$. When there is no adsorption of the surfactant at the substrate $\left(k_{a s}=0\right)$ the spreading is relatively slow (and we return to the situation shown in figures 2 and $4 f$ with the edge growing as $t^{1 / 7}$ ). For non-zero values of $k_{a s}$ the 

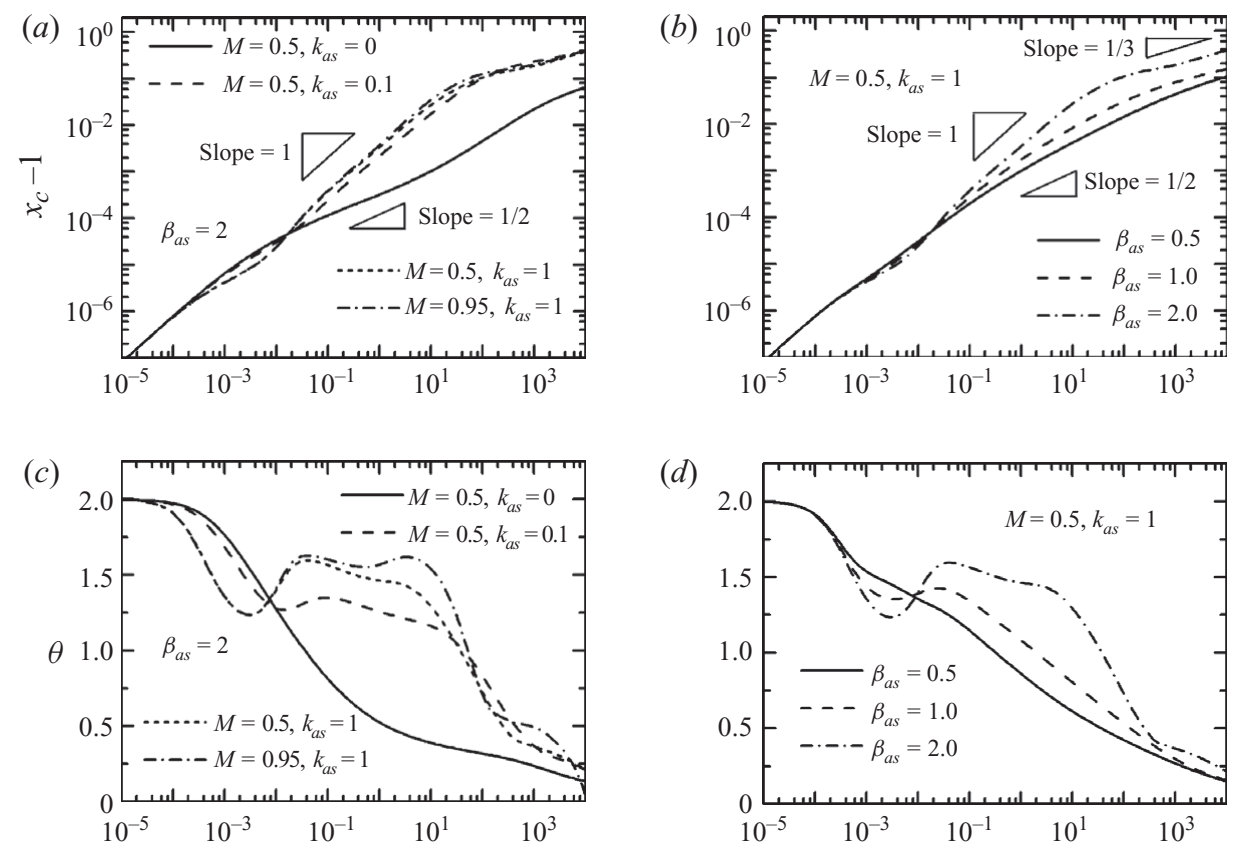

(d)
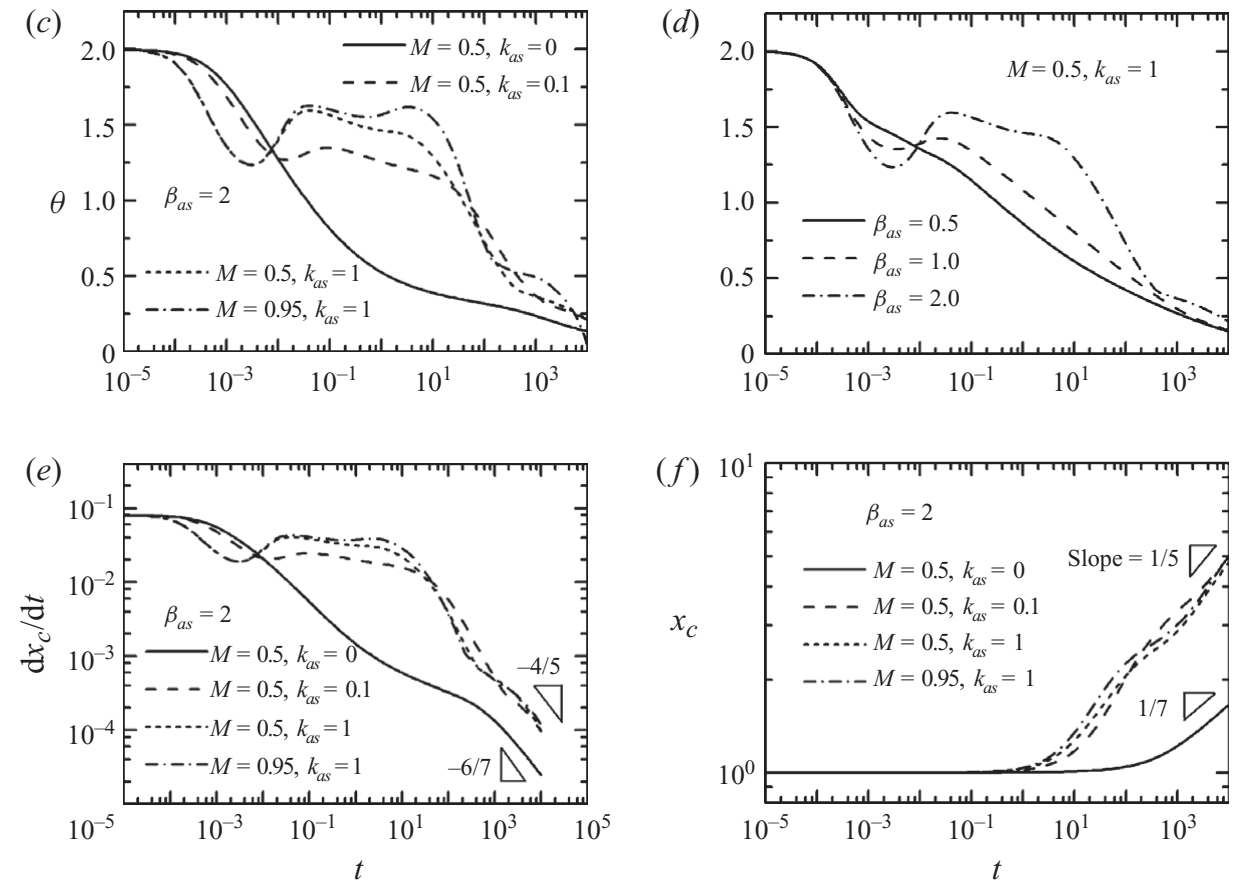

FIGURE 4. Effect of the parameters $M, k_{a s}$ and $\beta_{a s}$ for an insoluble surfactant with $\left(P e_{c a}, P e_{c s}\right)=\left(10^{4}, 10^{4}\right)$ on the time evolution of $(a, b) x_{c}-1,(c, d)$ the contact angle, $(e)$ the contact line velocity and $(f) x_{c}$.

spreading becomes dramatically faster, and the front in this case advances over several decades with almost a power of $t$. The key difference between these two cases is that in the first case the surfactant accumulates at the contact line, since it cannot go anywhere once it is there, whereas in the second case the surfactant is removed from the liquid-air interface as it is adsorbed at the substrate though the contact line, creating a local Marangoni stress. In order to get rapid spreading rates it is essential to prevent accumulation of the surfactant at the contact line, and this is achieved by adsorption of the surfactant at the solid surface, reducing surfactant concentrations to induce a Marangoni flow that promotes spreading. Another interesting observation is that increasing the value of $k_{a s}$ tenfold, from $k_{a s}=0.1$ to $k_{a s}=1$, has surprisingly little effect on the spreading rate. Even low values of the kinetic parameter $k_{a s}$ are capable of preventing surfactant accumulation. We observe, though, that at early stages the spreading rate is faster for $k_{a s}=1$, since the removal of the interfacial surfactant 

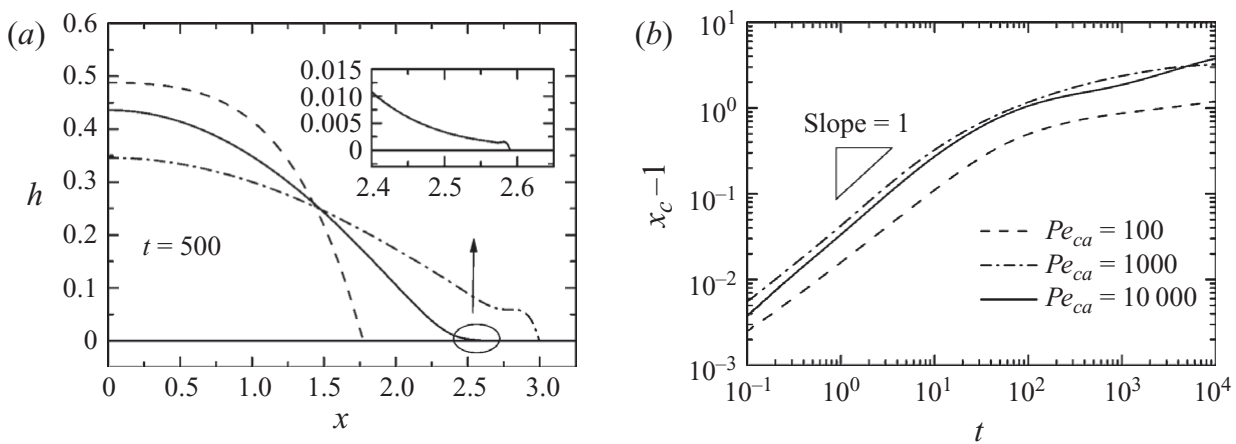

Figure 5. Effect of the Péclet number $P e_{c a}$ on the drop profile and the spreading rate of an insoluble surfactant with $\left(M, \beta_{a s}, k_{a s}, P e_{c s}\right)=\left(0.5,2,1,10^{4}\right)$.

is faster, and therefore the surface tension gradients in the liquid-air interface are higher, leading, in turn, to greater Marangoni stresses. However, this rapid adsorption depletes the interfacial surfactant, and thereafter the spreading rate becomes lower than for $k_{a s}=0.1$. Moreover, in this figure we can also see that the effect of the initial surfactant concentration is not very significant. The effect of $\beta_{a s}$ as presented in figure $4(b)$ is more important; the parameter $\beta_{a s}$ provides a dimensionless measure of the surfactant's ability to be adsorbed at the solid substrate $\left(\beta_{a s} \gg 1\right.$ signifies a very strong preference of the surfactant to be adsorbed at the solid substrate, and for $\beta_{a s} \ll 1$ it is virtually trapped upon the liquid-air interface). It is clear from the figure that the spreading rate increases as we increase the ability of the surfactant to be adsorbed at the substrate, which is in line with the thesis that it is adsorption that drives the higher spreading rates.

The droplet radius shown in figure $4(a, b)$ can, over some regimes, grow with a power-law exponent of order $t$ (spreading rate of order unity) rather than simply $t^{1 / 7}$. In figure $4(a, b)$ we show the droplet leading edge $x_{c}$ versus time by using $x_{c}-1 \sim t^{\alpha}$, where $t^{\alpha}$ is the power law that characterizes the leading-edge position with time, relative to its initial position. This is convenient, as, provided $x_{c} \gg 1$ for large time, this characterizes the long-time behaviour as $x_{c} \sim t^{\alpha}$. This also allows us to capture both short- and long-time scalings on a single graph: if the droplet grows very slowly, as in the insoluble, no-basal-adsorption case, then $x_{c}=O(1)$ over the time scale of the simulations, and figure 4(a) does not capture the long-time behaviour clearly; the $t^{1 / 7}$ scaling for the leading edge is therefore illustrated in figure $4(f)$ by using just $x_{c}$. A more transparent alternative is to plot the spreading rate explicitly as in figure $4(e)$, which clearly shows that the velocity of the droplet radius is approximately constant over the decades in time where $x_{c}-1 \sim O(t)$. It is remarkable how much higher the spreading rate for the cases with adsorption is versus that for cases without it (see figure $4 e$ ).

\subsubsection{Effect of surfactant diffusion along the liquid-air interface $\left(P e_{c a}\right)$}

Naturally interfacial diffusion of the surfactant could also play a role through damping the gradients of surface tension; the diffusion is measured by the Péclet number, and the effect of its variation is shown in figure $5(a, b)$ in which we present the drop profiles for $t=100$ and spreading rates for various values of the Péclet number $P e_{c a}$ respectively. The rest of the parameters, $M=0.5, k_{a s}=1, \beta_{a s}=2$ and $P e_{c s}=10^{4}$, allow comparison with figure 4 . For low values of $P e_{c a}$ diffusion smooths the surfactant concentration gradients close to the contact line, reducing the Marangoni stresses 
and therefore decreasing the spreading rate; the lowest spreading rate is found for $P e_{c a}=10^{2}$. Increasing the Péclet number to $P e_{c a}=10^{3}$ means that more surfactant is convected in the vicinity of the contact line. If adsorption at the contact line is rapid, then the surfactant concentration gradient becomes steeper, resulting in higher Marangoni stresses and, consequently, higher spreading rates. There is an interplay of physics here, as further increase of the Péclet number $\left(\mathrm{Pe}_{c a}=10^{4}\right)$ decreases the spreading rate because the rate of adsorption is not fast enough to remove the surfactant convected to the contact line.

\subsection{Soluble surfactant}

We now examine the spreading of a soluble surfactant at concentrations below, as well as beyond, the $\mathrm{CMC}$. The base case is now complemented by additional parameters, which were not required in the insoluble case, with typical values $M=5, \beta_{a}=1$, $\beta_{s}=2, k_{a}=k_{s}=k_{b}=k_{a s}=1, R_{a}=R_{s}=10, R_{a s}=1, N=10, P e_{c a}=P e_{c s}=10^{4}$. This set of parameters refers to a soluble surfactant with a concentration well above the CMC, which can exist as a monomer at the liquid-air, liquid-solid and solid-air interfaces as well as micellar aggregates in the bulk.

\subsubsection{Base case}

The evolutions of the drop and surfactant concentration profiles with time for the base case are shown in figure 6 . The surfactant concentration at the liquid-air interface, shown in figure $6(b)$, remains almost constant along the interface except for a region very close to the contact line where it decreases owing to the adsorption of the surfactant to the substrate at the contact line, which induces a Marangoni flow that promotes spreading. At early times, the Marangoni stresses tend to deform the free surface. The high surface tension very close to the contact line, however, provides large resistance to the deformation of the interface, and as a result, the contact angle retains relatively high values; this leads to the formation of a small raised rim that grows as the drop spreads out. The formation of such rims has also been observed experimentally (Rafai et al. 2002). We should note here that similar rims were also found in the spreading of surfactant-laden drops above the $\mathrm{CMC}$ over thin liquid substrates, where the rims we observe here are the counterpart of the secondary fronts observed in Edmonstone et al. (2006). Unlike the insoluble case in which the surfactant is adsorbed only at the solid substrate through the contact line, soluble surfactant monomers can also be adsorbed directly from the bulk, resulting in very different profiles of $c_{s}$, shown in figure $6(c)$. The surfactant concentration at the solid substrate, $c_{s}$, is maximal at the plane of symmetry and decreases as we approach the contact line, as the contact line continuously moves to surfactant-free regions. Of course, we should note here that, although some surfactant present at the contact line diffuses ahead of it, the diffusion is very weak, since the Péclet number is large, $P e_{c s}=10^{4}$, thus resulting in an abrupt decrease of $c_{s}$ ahead of the contact line (see figure $6 c$ ). The dilation of the liquid-air and liquid-solid interfaces, due to the fast spreading of the drop, causes the continuous decrease of the corresponding surfactant concentrations. As time passes, more and more surfactant monomers present in the bulk are adsorbed at the interfaces, and as a result the corresponding bulk concentration $c$ also decreases with time. This, in turn, leads the micelles to disassociate into monomers, and the micelle concentration decreases very rapidly. Note that the corresponding figure (see figure $6 e$ ) is presented only for early times $(t \leqslant 50)$, since after that point the concentration of micelles is very small; the 

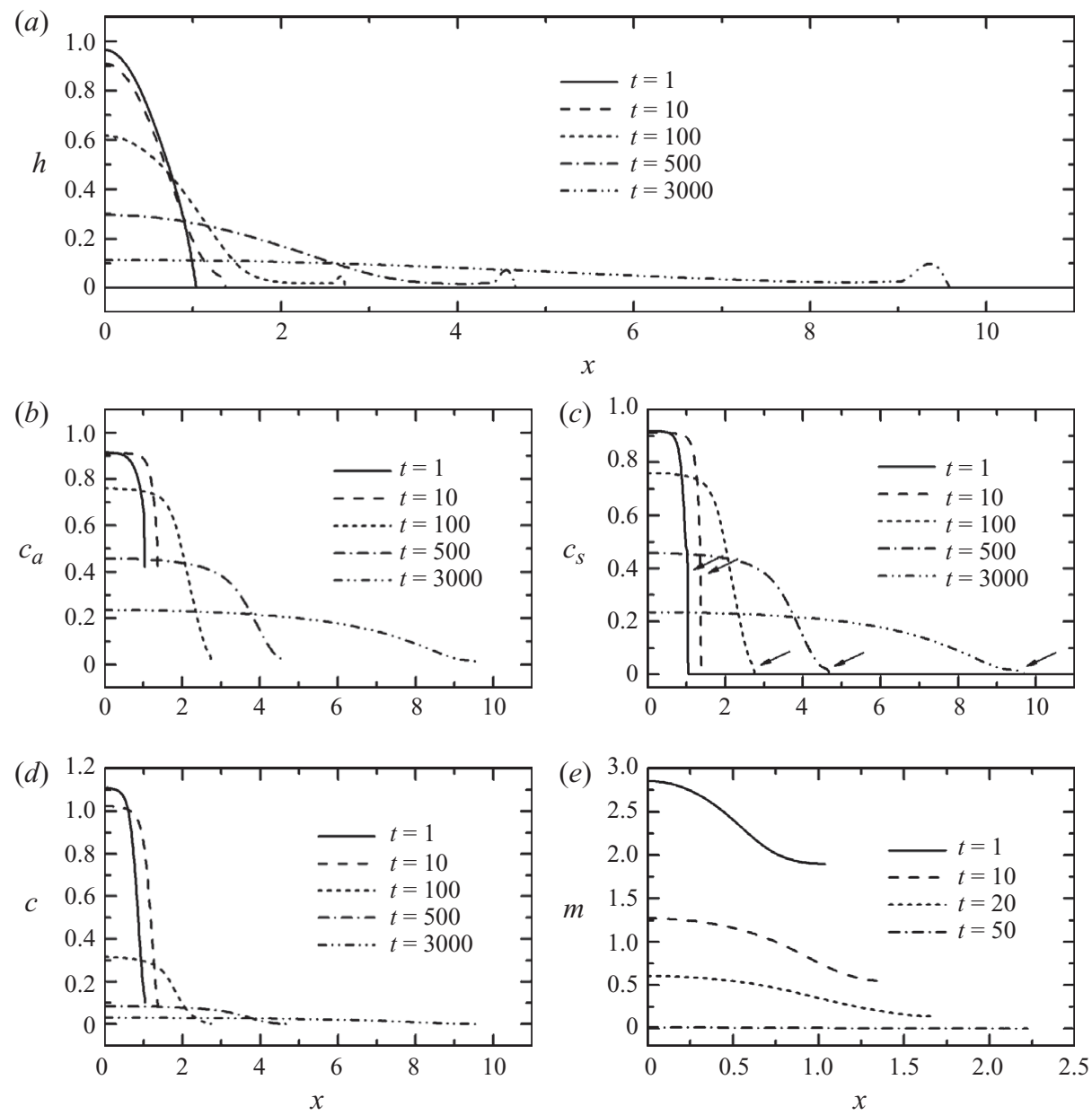

FIGURE 6. Time evolution of the drop profile and the surfactant concentrations for the base case of a soluble surfactant. These parameters are kept unchanged in the subsequent figures unless noted otherwise. The arrows in $(c)$ indicate the position of the contact line.

micelles act as a large reservoir releasing surfactant monomers and maintaining rapid spreading.

Very recently, Beacham et al. (2009) also presented a numerical study of a surfactant-laden drop spreading on a solid surface which also predicts the formation of a rim. A major difference with the present study is that they did not account for the explicit presence of a contact line and instead used a precursor model. Therefore, a key difference is that their model does not allow for the direct adsorption of the surfactant through the contact line, as is the case in our simulations shown in figure 6 . However, the presence of the precursor film can play a role similar to adsorption at the contact line, since the surfactant at the liquid-air interface in the region of the contact line can dissolve in the fluid that lies in the precursor film and, in turn, diffuse along it or be adsorbed at the substrate. Consequently, even in the model of Beacham et al. (2009) there is a mechanism for surfactant removal from the effective contact line, leading to the Marangoni stresses which are essential for very rapid spreading. 

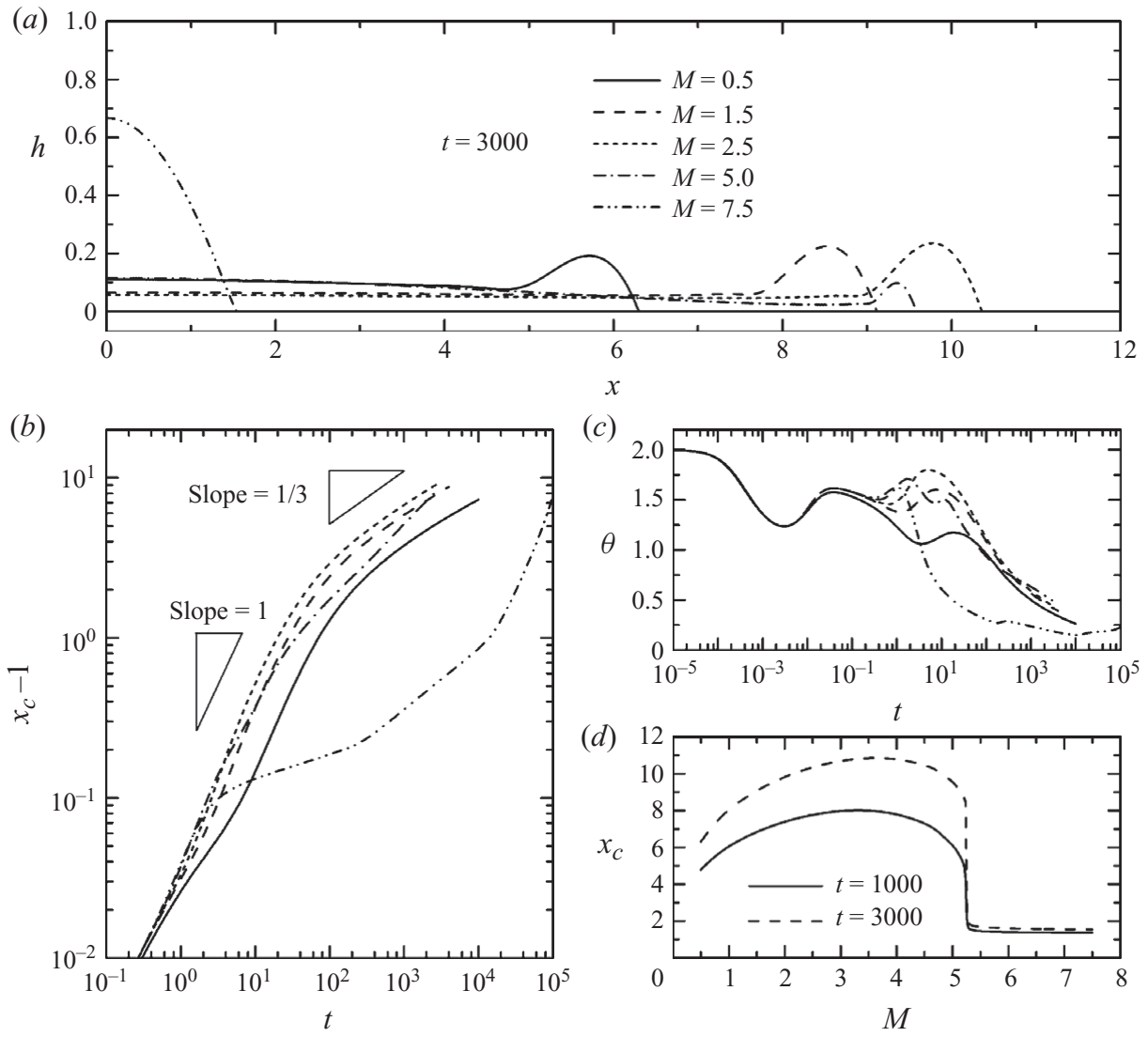

FIGURE 7. Effect of the initial surfactant concentration $M$ on $(a)$ the drop profile, $(b)$ the spreading rate, $(c)$ the time evolution of the contact angle for a soluble surfactant and $(d)$ the contact line position.

\subsubsection{Initial surfactant concentration}

Figure $7(a)$ presents the dependence of the drop profile at a given time instant $(t=3000)$ on the initial surfactant concentration $M$, and we see that the Marangoni stresses near the contact line have a significant effect on the drop profile even when $M$ is below the CMC $(M=0.5)$ and create a large rim. For $M=0.5$ the position of the contact line shows that the spreading rate is relatively slow, as there is not sufficient surfactant mass to maintain a significant Marangoni stress over a long time. As shown in figure $7(a)$, the extent of spreading at a given time instant, as well as the size of the rim, has a dependence on $M$ that is non-monotonic. Increasing $M$ up to $M=2.5$ results in increasing spreading rates as well as increasing size of the rim. However, further increase of $M$ results in the formation of smaller rims (e.g. for $M=5$ ) or even in a significant retardation of the spreading process for $M=7.5$.

The spreading rates can be seen more clearly in figure $7(b)$. In some of the cases presented here the front advances with a power almost equal to $t$, a rate in excess of $t^{2 / 3}$ predicted by the numerical modelling in Beacham et al. (2009). Interestingly, these spreading rates are very close to those reported by several experimentalists who have studied the effect of superspreading (e.g. see Nikolov et al. 2002; Rafai et al. 2002). It is noteworthy that even when there are no micelles present $(M=0.5)$ the 

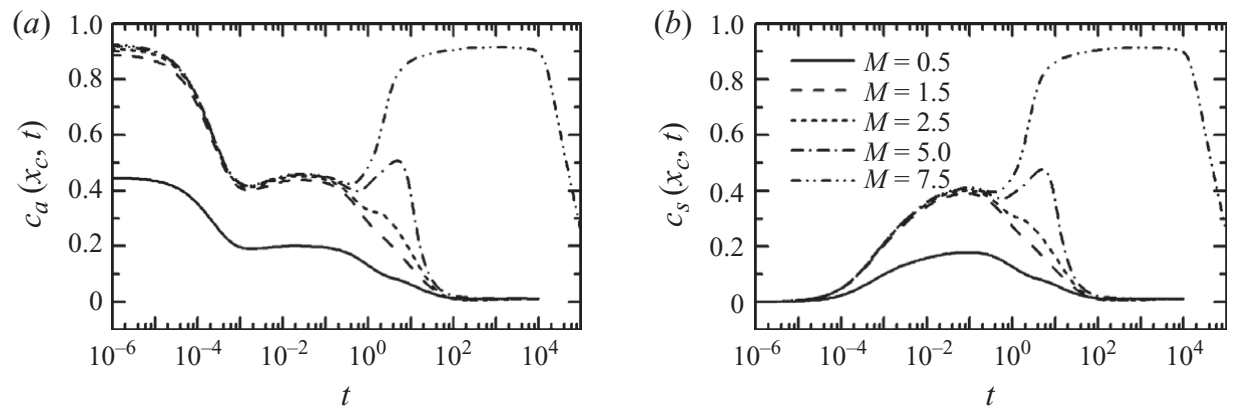

FIGURE 8. Effect of the initial surfactant concentration $M$ on the surfactant concentration at the contact line for a soluble surfactant.

spreading rate remains high for a significant amount of time. The issue of whether the presence of micelles is necessary for superspreading was studied by Nikolov et al. (2002), and their experiments have shown that fast spreading is possible even in the absence of micelles, in agreement with our findings. The presence of the rim at the leading edge plays an important role in obtaining high spreading rates, since its convex shape results in high contact angles, which are maintained for a long period. This can be seen very clearly in figure $7(c)$ where we present the evolution of the contact angles with time for various values of $M$. The dependence of the contact angle on time is rather complex, but we can observe that the spreading rate is high as long as the contact angles remains high. As noted earlier, the dependence of the spreading rates on the initial surfactant concentration $M$ is non-monotonic, which is also true for $h(0, t)$. It is interesting that the spreading associated with $M=7.5$, which is initially fast, after some point in time becomes rather slow and accelerates again at later times. The long-time $t^{1 / 3}$ scaling for droplet spreading follows from a Marangoni-dominated regime, such as that found for constant effectively insoluble surfactant mass transport on a monolayer as in Jensen \& Grotberg (1993), and suggests that once the micellar and bulk surfactant reservoir has become depleted, the droplet spreads purely under the action of surface tension gradients across the whole droplet rather than those localized at the edge. The non-monotonic variation of the spreading rates is shown clearly in figure $7(d)$ and has also been reported in numerous experimental works (e.g. see Zhu et al. 1994; Stoebe et al. 1996; Nikolov et al. 2002) as well as the numerical study by Beacham et al. (2009). A possible explanation for this non-monotonic behaviour is given herein. In our model, the Marangoni stresses, which cause the fast spreading of the drop, are maintained by the continuous removal of the surfactant from the liquid-air interface by adsorption at the solid substrate. On the other hand, we know that the surfactant tends to increase locally at the contact line, as it is transferred there by diffusion and advection along the free surface, by adsorption of the monomers in the bulk at the liquid-air interface or even by desorption directly from the substrate at the contact line. Of course, increasing the initial surfactant concentration $M$ would make this transport even more significant. If the rate of adsorption at the contact line is sufficiently rapid, then increasing $M$ leads to higher gradients of $c_{a}$ and thus to higher Marangoni stresses. However, after some point, the rate of adsorption is simply not fast enough, and this results in lower Marangoni stresses, leading, in turn, to smaller spreading rates. This is seen clearly in figure 8 in which we present the effect of $M$ on the surfactant concentrations at the contact line. For $M \leqslant 3.5$ the rate of adsorption is 
enough to result in a continuous decrease of the interfacial surfactant concentration at the contact line. However, for larger values of $M$ the surfactant accumulates at the contact point, resulting in the decrease of the spreading rate. It is characteristic that for $M=7.5$ the surfactant concentration remains large for a large amount of time, resulting in small spreading rates. The increase in the spreading rate at later times could be attributed to the fact that during the phase of slow spreading there is enough time for the surfactant to diffuse ahead of the contact line, which eventually results in the decrease of the surfactant concentration at the contact line, again inducing strong Marangoni stresses that re-initiate the fast spreading process. Therefore, it becomes evident that the non-monotonic behaviour is due to the interplay of the rate of transport towards the contact line of surfactant monomers at the liquid-air interface and the rate of their removal.

\subsubsection{Effect of basal adsorption of surfactant}

From our results so far basal adsorption of the surfactant is seen to play a crucial role in the spreading process. In order to investigate its effect further we consider the effect of the parameter $\beta_{s}$ which controls the amount of the surfactant that can be adsorbed at the solid substrate; as shown in figure $9(d)$, its variation affects considerably the spreading rates of the drop. More specifically, increasing the value of $\beta_{s}$, which means that more surfactant can be adsorbed at the substrate, results in a significant increase of the spreading rate. At moderate times and for the highest value of $\beta_{s}\left(\beta_{s}=5\right)$ the spreading rate is extremely fast, and the front advances with a power even higher than $t$. However, the fast spreading of the drop soon leads to the depletion of the surfactant that lies upon the liquid-air interface (see figure $9 e$ ), and after that point the spreading rate starts to decrease. On the other hand, the spreading rate for $\beta_{s}=2$ does not change significantly with time, and the front advances with a power almost equal to $t$ throughout the simulation. Even though the spreading rate for $\beta_{s}=5$ initially is higher than for $\beta_{s}=2$ the long-time extent of spreading for the latter is larger because there is more surfactant available to be adsorbed at the liquidair interface, and therefore the spreading rate remains almost constant for a longer period. For the lowest value of $\beta_{s}$ shown, $\beta_{s}=0.5$, the droplet leading edge advances as $x_{c}-1 \sim O\left(t^{1 / 2}\right)$, and the $1 / 2$ scaling is consistent with a droplet advancing because of the presence of a surfactant reservoir, as in Edmonstone et al. (2006), suggesting that the adsorption is not rapid enough to create the local Marangoni gradients at the edge necessary for rapid spreading.

The drop profiles for the same values and at $t=3000$ are also presented in figure $9(a)$ for different values of $\beta_{s}$. At that time instant, the extent of spreading for the low values of $\beta_{s}$ is quite small because of the decreased spreading rates, whereas for $\beta_{s} \geqslant 2$ the drop has wetted a much larger area. The spreading in the latter case is accompanied by the formation of a rim at the advancing front, the size of which increases significantly with $\beta_{s}$. It is characteristic that for $\beta_{s}=5$, the largest value of $\beta_{s}$ investigated, the rim contains almost the entire volume of the drop. The increase of $\beta_{s}$ signifies that more surfactant can be adsorbed at the solid substrate before it becomes saturated. This can be seen very clearly in figure $9(c)$ in which we present for $t=1$ the dependence of $c_{s}$ on $\beta_{s}$. Note that at this time instant there is no formation of a rim, and the extent of spreading is similar in all cases (the arrows in the figure indicate the position of the contact line). For the lowest value of $\beta_{s}\left(\beta_{s}=0.5\right)$ the concentration at the substrate, inside the drop, is almost constant and very close to its saturation value, except for a region very close to the contact line. The latter is due to the fact that the drop continuously spreads into uncontaminated areas of the substrate. Increasing $\beta_{s}$, 
(a)

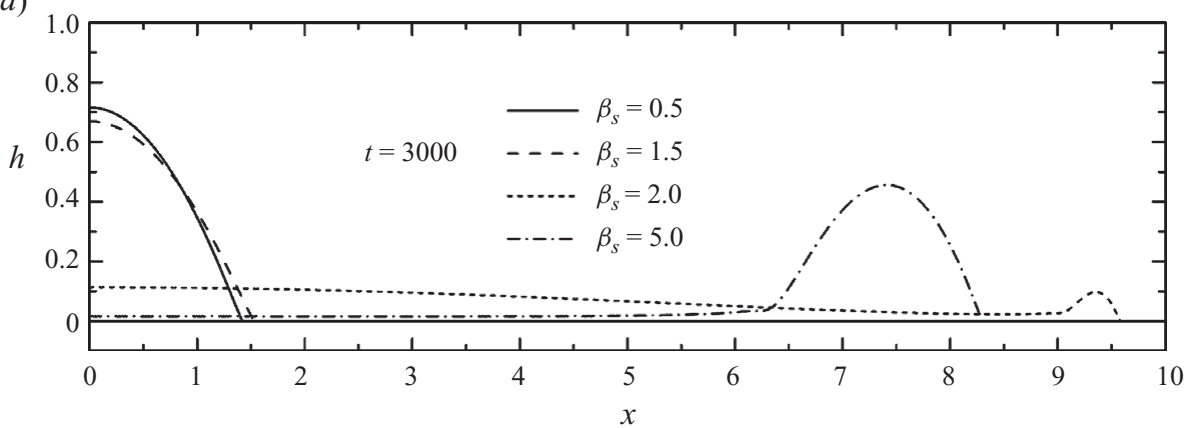

(b)
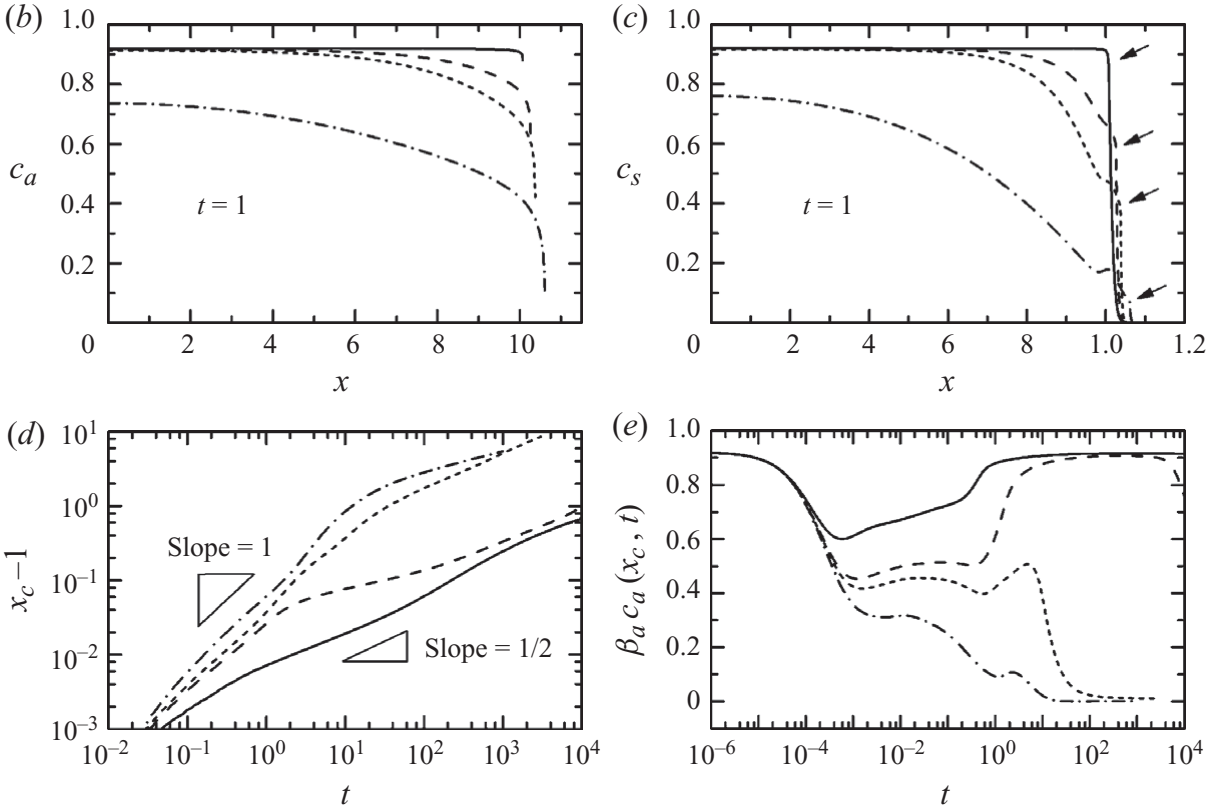

FIGURE 9. Effect of the parameter $\beta_{s}$ on $(a)$ the drop profile, $(b, c)$ the interfacial concentration profiles (the arrows indicate the position of the contact line), $(d)$ the spreading rate and $(e)$ the surfactant concentrations at the contact line for a soluble surfactant.

however, results in the considerable decrease of $c_{s}$, and consequently more surfactant monomers in the bulk can be adsorbed at the substrate at later times. The decrease of the amount of monomers in the bulk which are available to be adsorbed at the liquid-air interface will result in the decrease of the rate of adsorption along the liquid-air interface and thus in the decrease of $c_{a}$. This is shown, clearly, in figure $9(b)$. Moreover, we observe that the increase of $\beta_{s}$ gives rise to higher concentration gradients, and these gradients act over a larger area along the liquid-air interface. We noted earlier that the rims are formed because of Marangoni stresses and that the high surface tension close to the contact line provides large resistance to the deformation of the interface. As is shown in figure $9(b)$ the increase of $\beta_{s}$ leads, at early times, to larger areas of high surface tension (low surfactant concentration) close to the contact line, and this will result eventually in the formation of a larger rim; these observations are also in qualitative agreement with the results presented in Beacham et al. (2009). Those authors also noted that decreasing the value of $R_{a}$ is qualitatively similar to increasing $\beta_{s}$, since low values of $R_{a}$ signify the preference of 

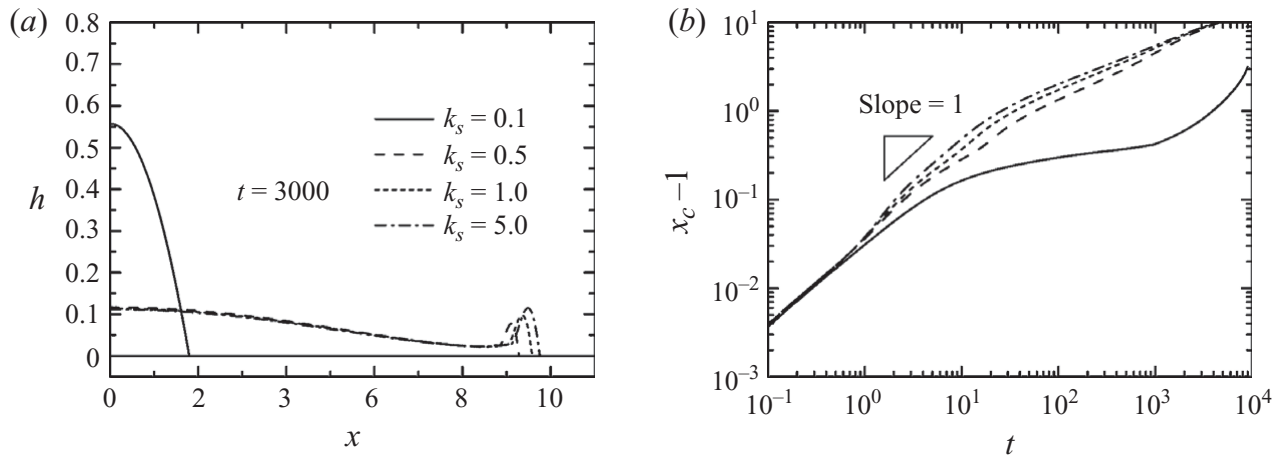

FIGURE 10. Effect of the parameter $k_{s}$ on $(a)$ the drop profile and $(b)$ the spreading rate for a soluble surfactant.

the surfactant to remain in the bulk than to be adsorbed at the liquid-air interface, and therefore the amount of the surfactant that is available to be adsorbed to the substrate increases. Similar behaviour is also found in our simulations but is not presented here for the sake of brevity.

Apart from the amount of the surfactant that can be adsorbed at the solid substrate, it is also important to examine the effect of the sorption kinetics. In this study, we have adopted a model which assumes that the surfactant is adsorbed at the solid surface with two different mechanisms. The first mechanism considers that the surfactant monomers in the bulk can be adsorbed/desorbed at the solid substrate, according to the kinetic law shown in (2.11). The second mechanism concerns the surfactant monomers that lie upon the liquid-air interface, which can be adsorbed/desorbed at the substrate directly through the contact line, according to the 'reaction' shown in (2.13). To start with, we present in figure 10 the effect of the sorption kinetics for the monomers in the bulk. This figure depicts the drop profiles at $t=3000$ as well as the spreading rates for various values of the kinetic parameter $k_{s}$. As shown in figure $10(a)$, for all but the lowest value of $k_{s}$, the drop has wetted a large area of the solid wall, and a rim has been formed at the leading edge. Decreasing the parameter $k_{s}$ results in the decrease of the rim size as well as in reduced spreading rates. When the sorption kinetics are very slow $\left(k_{s}=0.1\right)$ the monomers in the bulk prefer to be adsorbed at the liquid-air interface, resulting in lower surface tension gradients and therefore in smaller spreading rates.

\subsubsection{Adsorption/desorption at the substrate through the contact line}

Here, we examine the effect of the adsorption of monomers that lie upon the liquidair interface at the contact line. Figure 11 presents the evolution of the drop profiles for three different values of the kinetic parameter $k_{a s}$. The effect of this parameter is also examined in figure 12 in which we present the spreading rates as well as the evolution of the drop thickness at the plane of symmetry $(x=0)$ with time. Clearly, from figure 11 we deduce that the sorption kinetics have a significant effect on the extent of spreading as well as the resulting shapes of the drops. More specifically, for $k_{a s}=0$ (no adsorption at the contact line) the spreading of the drop is relatively slow, and its shape at the end of the simulation $\left(t=2.5 \times 10^{5}\right)$ resembles a thick 'pancake'. Since in this case there is no adsorption at the contact line, the only way for a monomer that lies at the liquid-air interface to be adsorbed at the substrate would be to dissolve first in the bulk fluid and then to be adsorbed at the solid wall, 

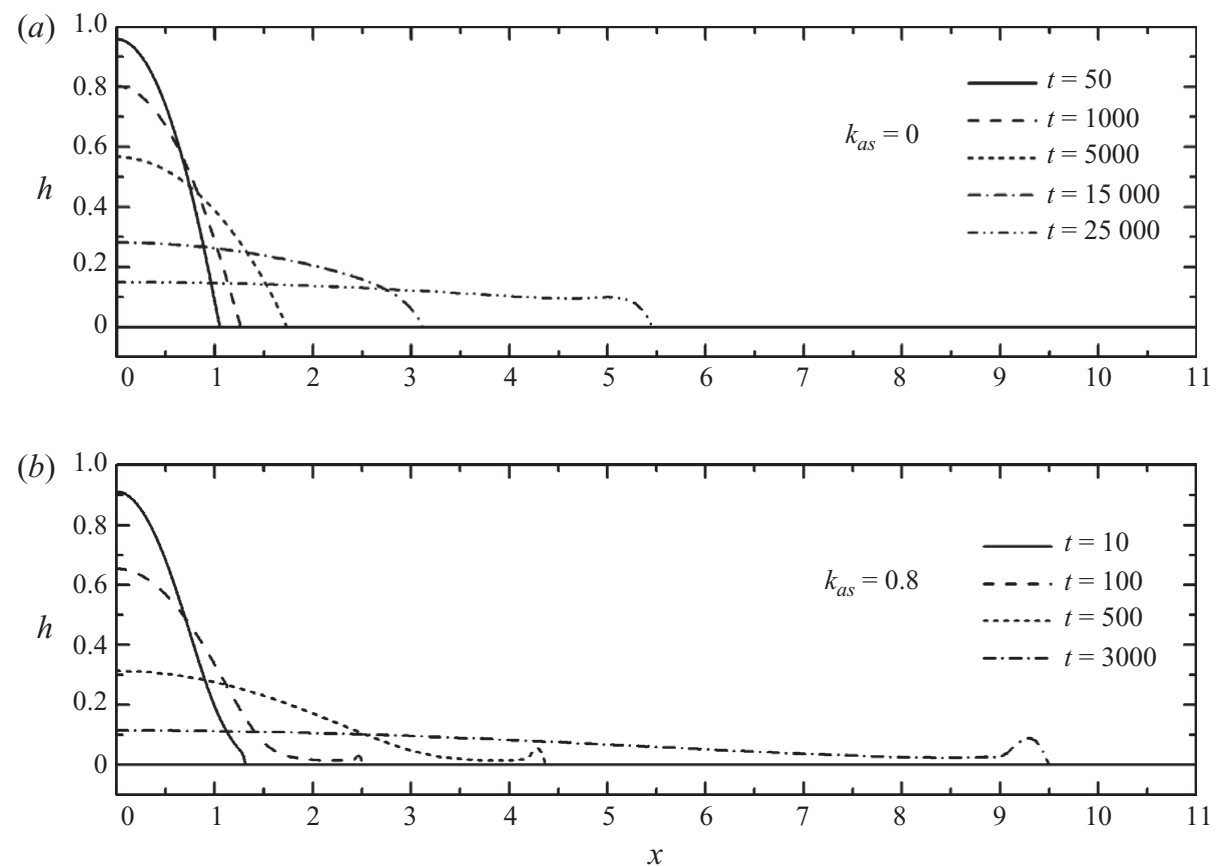

FIGURE 11. Time evolution of the drop profiles with time for various values of $k_{a s}$ for a soluble surfactant.
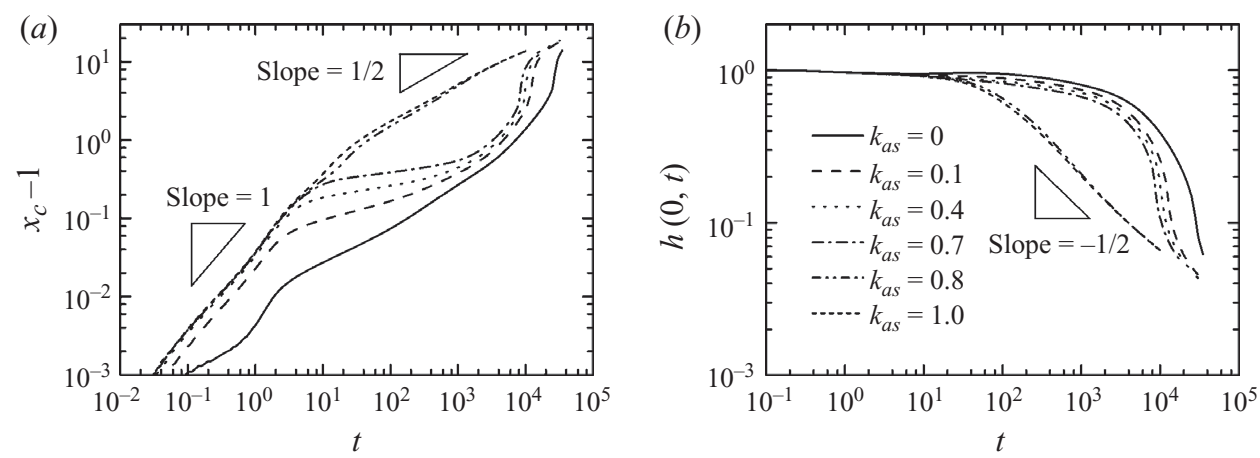

FIGURE 12. Effect of the parameter $k_{a s}$ on $(a)$ the spreading rate and $(b)$ the evolution of the drop thickness at the plane of symmetry for a soluble surfactant.

as described above. In this case, the removal of surface monomers from the region close to the contact line becomes, obviously, more difficult. Eventually, this results in smaller surface tension gradients at the free surface and thus lower Marangoni stresses near the leading edge that are not able to promote fast spreading.

For finite $k_{a s}$, the situation becomes somewhat more complicated. When adsorption at the contact line is fast enough (for $k_{a s} \geqslant 0.8$ ) the surfactant is constantly removed from the liquid-air interface close to the contact line, leading to strong Marangoni forces that maintain fast spreading at all times. However, for intermediate values of $k_{a s}$ $\left(0<k_{a s} \leqslant 0.7\right)$ the spreading can be divided largely into four different regimes. During the early stages of the spreading process, the spreading rate is high regardless of the value of $k_{a s}$. After some time, however, the spreading rate decreases significantly. 

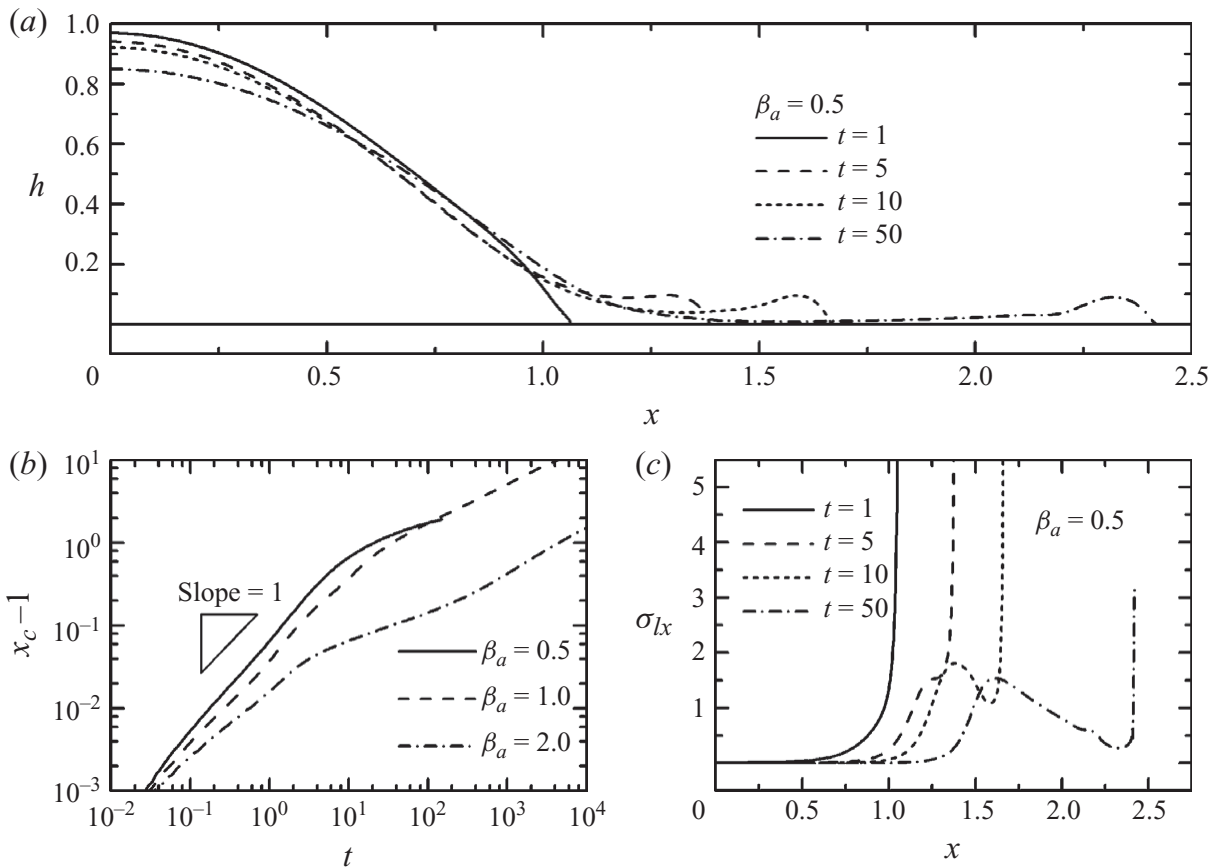

FIGURE 13. ( $a$ ) Time evolution of the drop profile with time for $\beta_{a}=0.5$. Effect of $\beta_{a}$ on the $(b)$ spreading rate and $(c)$ surface tension gradient for a soluble surfactant.

This happens because the rate of adsorption at the contact line is not fast enough to maintain the surface gradients on the liquid-air interface, and as a result, the Marangoni stresses cannot maintain for long the rapid spreading of the drop. The slow spreading of the drop is maintained for quite some time, followed by a sudden collapse of the drop. A possible explanation for this behaviour is that during the phase of slow spreading there is enough time for the surfactant to diffuse ahead of the contact line, which eventually results in the decrease of the surfactant concentration at the contact line, again inducing strong Marangoni stresses that re-initiate the fast spreading process. When, however, the surfactant is depleted the spreading reverts to the same limit as for higher values of $k_{a s}$.

\subsubsection{Surfactant solubility}

The effect of surfactant solubility for varying values of the parameter $\beta_{a}\left(\beta_{a} \ll 1\right.$ signifies high solubility, whereas for $\beta_{a} \gg 1$ the surfactant is virtually insoluble and is trapped upon the free surface) is examined in figure 13. Decreasing the value of $\beta_{a}$, while maintaining a fixed mass of the surfactant, leads to a decrease in the total mass of the surfactant that can be adsorbed at the liquid-air interface, which means that more surfactant is adsorbed at the substrate; this is qualitatively similar to increasing the value of $\beta_{s}$. This relation can be seen clearly by comparing figures $9(d)$ and $13(b)$, where the spreading rates are presented for various values of $\beta_{a}$ and $\beta_{s}$, respectively. We observe that the curve for $\beta_{a}=0.5$ stops at relatively early times. This is because the simulation for this case ends quite early, and the reason for this can be seen in figure 13(a) which shows how the drop profile evolves with time. At early stages, the Marangoni flow deforms the free surface in the vicinity of the contact line, and a rim is formed. However, later on, the rim splits away from the 
main drop to form an isolated front separated from the main droplet. The surface gradients in the middle of the region that connects the ridge at the leading edge and the main droplet increase significantly with time (see figure 13c), resulting in increased Marangoni stresses, which drive the fluid that resides there towards the contact line. These regions can eventually become so thin that they may appear as 'dry spots' when viewed by an experimental observer. In the experiments of Nikolov et al. (2002), who studied the spreading of a TS8EO solution placed on a polystyrene surface, the separation of small drops from the leading edge of the main droplet at the final stages of the spreading process was reported. Moreover, Edmonstone et al. (2006), who performed numerical simulations on the spreading of a droplet of soluble surfactant on a pre-existing thin liquid layer, showed a similar formation of a 'secondary' front and exceptionally thin regions.

\section{Concluding remarks}

We have examined the spreading of surfactant-laden droplets over solid substrates for both insoluble and soluble surfactants with concentrations below, as well as beyond, the CMC. Lubrication theory and rapid vertical diffusion of the surfactant in the bulk have been used to derive a coupled system of evolution equations for the drop thickness, surfactant monomer interfacial and bulk concentrations and micelle bulk concentration. The model accounts for Marangoni-driven spreading, interfacial and bulk diffusion, sorption kinetics, the formation and breakup of micelles in the bulk and surfactant adsorption at the solid substrate. Moreover, this model takes into account the moving contact line, using a constitutive relation that depends on the local surfactant concentration and the possibility of the adsorption of the surfactant that lies upon the liquid-air interface at the solid surface directly through the contact line. This model has been numerically solved using the finite-element method, and an extensive parametric analysis has been presented.

The key issue in terms of superspreading and surfactant-enhanced spreading is the identification of a mechanism by which high spreading rates can be achieved. The model derived above is sufficiently complex to allow most of the relevant physics and chemistry to be incorporated while simple enough to allow for interpretation. Our numerical investigation has shown that basal adsorption (from the bulk fluid and at the contact line) as well as sorption kinetics play a crucial role in the spreading process. This physics can provide an effective description of the molecular-scale picture of the adsorption of the superspreading trisiloxane surfactants. When the trisiloxanes are dissolved in an aqueous phase, their structure allows them to be adsorbed onto a hydrophobic surface because they can pack tightly as a hydrophobic mat on the non-polar surface (see figure 1) - with the polar groups exposed to the aqueous phase. In this tight packing, the hydrophobic interior of the mat is not exposed to water. Further, at the contact line, it appears that monolayers at the air-liquid and solid-liquid interfaces come together and assemble to form a bilayer, and hence the assumption of the direct adsorption from the air/liquid surface to the contact line is sensible. To get efficient spreading it is essential to have a balance of surfactant replenishment at the liquid-air interface and its removal close to the contact line so that there are locally high surface tension gradients and therefore high Marangoni stresses to generate fast spreading. The incorporation of these two adsorption routes provides such a mechanism. This forms the link to understanding superspreading; other surfactants are not adsorbed significantly from the aqueous solution onto a hydrophobic surface and do not assemble into bilayers at the contact line. 
Our numerical results have shown that the velocity of the droplet leading edge is constant with the extent of the leading edge being characterized by power laws of $t^{a}$ with $a=1$ or even higher, which are close to the reported experimental values for superspreading. There is, however, significant dependence of the calculated spreading rates on the values of the various kinetic parameters. In addition, the non-monotonic dependence of the spreading rate on the initial surfactant concentration, often reported in experiments, has been predicted (see figure 7). The spreading is accompanied in several cases with the generic formation of a rim, which under certain conditions may contain almost the entire volume of the drop (see figure 9). The parametric study has revealed that this is not always the case. For example, when adsorption of the surfactant at the contact line is not allowed the resulting shape of the drop resembles a thick pancake (see figure 11). Finally, when the solubility of the surfactant is relatively high a secondary front separated from the main droplet may appear (see figure 13). The region in between can become so thin that it may appear as 'dry spots' when viewed by an experimental observer.

The insightful comments of one anonymous referee, regarding the physicochemical phenomena that underlie superspreading, are gratefully acknowledged. The authors also acknowledge the support of the Engineering and Physical Science Research Council through grant number EP/E056466.

\section{REFERENCES}

Beacham, D. R., Matar, O. K. \& Craster, R. V. 2009 Surfactant-enhanced rapid spreading of drops on solid surfaces. Langmuir 25, 14174-14181.

Benintendi, S. W. \& Smith, M. K. 1999 The spreading of a non-isothermal liquid droplet. Phys. Fluids 11, 982-989.

Bonn, D., Eggers, J., Indekeu, J., Meunier, J. \& Rolley, E. 2009 Wetting and spreading. Rev. Mod. Phys. 81, 739-805.

Carlson, A., Do-Quang, M. \& Amberg, G. 2009 Modelling of dynamic wetting far from equilibrium. Phys. Fluids 21, 121701-121704.

Chan, K. Y. \& Borhan, A. 2005 Surfactant-assisted spreading of a liquid drop on a smooth solid surface. J. Colloid Interface Sci. 287, 233-248.

Clay, M. A. \& Miksis, M. J. 2004 Effects of surfactant on droplet spreading. Phys. Fluids. 16, 3070-3078.

Cox, R. G. 1986a The dynamics of the spreading of liquids on a solid surface. Part 1. Viscous flow. J. Fluid Mech. 168, 169-194.

Cox, R. G. $1986 b$ The dynamics of the spreading of liquids on a solid surface. Part 2. Surfactants. J. Fluid Mech. 168, 195-220.

Craster, R. V. \& Matar, O. K. 2006 On the dynamics of liquid lenses. J. Colloid Interface Sci. 303, 503-516.

Craster, R. V. \& MataR, O. K. 2007 On autophobing in surfactant-driven thin films. Langmuir 23, $2588-2601$.

Dussan, V. \& Davis, S. H. 1974 On the motion of a fluid-fluid interface along a solid surface. J. Fluid Mech. 65, 71-95.

Dussan, V., RAME, E. \& GAROFF, S. 1991 On identifying the appropriate boundary conditions at a moving contact line: an experimental investigation. J. Fluid Mech. 230, 97-116.

Edmonstone, B. D., Craster, R. V. \& Matar, O. K. 2006 Surfactant-induced fingering phenomena beyond the critical micelle concentration. J. Fluid Mech. 564, 105-138.

Edmonstone, B. D., Matar, O. K. \& Craster, R. V. 2005 Surfactant-induced fingering phenomena in thin film flow down an inclined plane. Physica D 209, 62-79.

Ehrhard, P. 1993 Experiments on isothermal and non-isothermal spreading. J. Fluid Mech. 257, 463-483. 
Ehrhard, P. \& Davis, S. H. 1991 Non-isothermal spreading of liquid drops on horizontal plates. J. Fluid Mech. 229, 365-388

Gaver, D. P. III \& GrotberG, J. B. 1990 The dynamics of a localized surfactant on a thin film. J. Fluid Mech. 213, 127-148.

DE Gennes, P. G. 1985 Wetting: statics and dynamics. Rev. Mod. Phys. 57, 827-863.

GreEnspan, H. P. 1978 On the motion of a small viscous droplet that wets a surface. J. Fluid Mech. 84 125-143.

Grotberg, J. B. 1994 Pulmonary flow and transport phenomena. Annu. Rev. Fluid Mech. 26, 529-571.

Haley, P. J. \& Miksis, M. J. 1991 The effect of the contact line on droplet spreading. J. Fluid Mech. 223, 57-81.

HiLl, R. M. 1998 Superspreading. Curr. Opin. Colloid Interface Sci. 3, 247-254.

Hill, R. M. 2002 Silicone surfactants-new developments. Curr. Opin. Colloid Interface Sci. 7, 255261.

Huh, C. \& Scriven, L. E. 1971 Hydrodynamic model of steady movement of a solid/liquid/fluid contact line. J. Colloid Interface Sci. 35, 85-101.

Hunter, R. J. 1991 Foundations of Colloid Science. Oxford University Press.

Jensen, O. E. \& Grotberg, J. B. 1993 The spreading of heat or soluble surfactant along a thin film. Phys. Fluids A 5, 58-68.

Jensen, O. E. \& NaIRE, S. 2006 The spreading and stability of a surfactant-laden drop on a prewetted substrate. J. Fluid Mech. 554, 5-24.

Kataoka, D. E. \& Troian, S. M. 1997 A theoretical study of instabilities at the advancing front of thermally driven coating films. J. Colloid Interface Sci. 192, 350-362.

Kim, H.-Y., Qin, Y. \& FichthoRn, K. A. 2006 Molecular dynamics simulation of nanodroplet spreading enhanced by linear surfactants. J. Chem. Phys. 125, 174708.

Knoche, M., Tamura, H. \& Bukovac, J. 1991 Performance and stability of the organosilicone surfactant L-77: effect of $\mathrm{pH}$, concentration, and temperature. J. Agric. Food Chem. 39, 202-206.

Kondic, L. \& Diez, J. 2001 Pattern formation in the flow of thin films down an incline: constant flux configuration. Phys. Fluids 13, 3168-3184.

Kumar, N., Couzis, A. \& Maldarelli, C. 2003 Measurement of the kinetic rate constants for the adsorption of superspreading trisiloxanes to an air/aqueous interface and the relevance of these measurements to the mechanism of superspreading. J. Colloid Interface Sci. 267, $272-285$.

Navier, C. L. M. H. 1823 Mémoire sur les lois du mouvement des fluides. Acad. R. Sci. Inst. Fr. 6, $389-440$.

Nikolov, A. D., Wasan D. T., Chengara, A., Koczo, K., Policello, G. A. \& Kolossvary, I. 2002 Superspreading driven by Marangoni flow. Adv. Colloid Interface Sci. 96, 325-338.

Radulovic, J., Sefiane, K. \& Shanahan, M. E. R. 2009 Spreading and wetting behaviour of trisiloxanes. J. Bionic Engng 6, 341-349.

Rafai, S., Sarker, D., Bergeron, V., Meunier, J. \& Bonn D. 2002 Superspreading: aqueous surfactant drops spreading on hydrophobic surfaces. Langmuir 18, 10486-10488.

RAME, E. 2001 The spreading of surfactant-laden liquids with surfactant transfer through the contact line. J. Fluid Mech. 440, 205-234.

SCHWARTZ, L. W. \& ElEy, R. R. 1998 Simulation of droplet motion on low-energy and heterogeneous surfaces. J. Colloid Interface Sci. 202, 173-188.

Sheludko, A. 1967 Thin liquid films. Adv. Colloid Interface Sci. 1, 391-464.

Spaid, M. A. \& Homsy, G. M. 1996 Stability of Newtonian and viscoelastic dynamic contact lines. Phys. Fluids 8, 460-478.

Stoebe, T., Hill, R. M., Ward, M. D. \& Davis, H. T. 1997 a Enhanced spreading of aqueous films containing ionic surfactants on solid substrates. Langmuir 13, 7276-7281.

Stoebe, T., Lin, Z., Hill, R. M., Ward, M. D. \& Davis, H. T. 1996 Surfactant-enhanced spreading. Langmuir 12, 337-344.

Stoebe, T., Lin, Z., Hill, R. M., Ward, M. D. \& Davis, H. T. $1997 b$ Enhanced spreading of aqueous films containing ethoxylated alcohol surfactants on solid substrates. Langmuir 13, 7270-7275.

Stoebe, T., Lin, Z., Hill, R. M., Ward, M. D. \& Davis, H. T. 1997c Superspreading of aqueous films containing trisiloxane surfactant on solid substrates. Langmuir 13, 7282-7286. 
TANnER, L. H. 1979 The spreading of silicone oil drops on horizontal surfaces. J. Phys. D 12, $1473-1484$.

Warner, M. R. E., Craster, R. V. \& Matar, O. K. 2004a Fingering phenomena created by a soluble surfactant deposition on a thin liquid film. Phys. Fluids 16, 2933-2951.

Warner, M. R. E., Craster, R. V. \& Matar, O. K. 2004b Fingering phenomena associated with surfactant spreading on thin liquid films. J. Fluid Mech. 510, 169-200.

Zhu, S. Miller, W. G., Scriven, L. E. \& Davis, H. T. 1994 Superspreading of water-silicone surfactant on hydrophobic surfaces. Colloids Surf. A 90, 63-78. 